\title{
Research Paper \\ The mediating role of achievement goals in the relationship between the personality trait of grit and academic engagement and procrastination among high school students: a structural model
}

Alireza Rouhi ${ }^{1}$, Javad Kavousian², Masoud Geramipour ${ }^{3}$, Hadi Keramati ${ }^{2}$, Mehdi Arabzadeh ${ }^{2}$

1. Ph.D Candidate in Educational Psychology, Educational Psychology Department, Faculty of Psychology and Educational Sciences, Kharazmi University of Tehran, Iran.

2. Assistant Professor, Educational Psychology Department, Faculty of Psychology and Educational Sciences, Kharazmi University of Tehran, Iran

3. Assistant Professor, Curriculum Planning Department, Faculty of Psychology and Educational Sciences, Kharazmi University of Tehran, Iran.

Citation: Rouhi A, Kavousian J, Geramipour M, Keramati H, Arabzadeh M. The mediating role of achievement goals in the relationship between the personality trait of grit and academic engagement and procrastination among high school students: a structural model. J of Psychological Science. 2022; 20(107): 19451964.

URL: https://psychologicalscience.ir/article-1-1297-fa.html
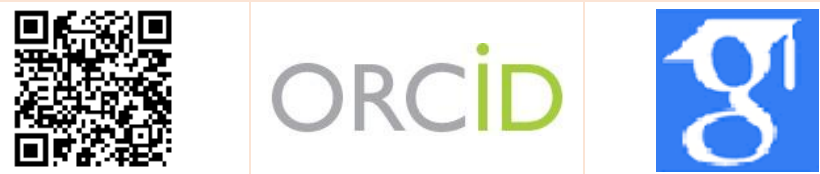

$\underline{10.52547 / J P S .20 .107 .1945}$

\section{A R T I C L E I N F O A B S T R A C T}

Keywords:

Grit,

Achievement goals, Academic engagement, Academic procrastination

Received: 12 Jun 2021 Accepted: 30 Jul 2021 Available: 21 Jan 2022
Background: Although previous researches have examined the direct effects of the personality trait of grit in predicting students' procrastination and engagement, its indirect effects have been less studied.

Aims: The general purpose of this study is to investigate the role of grit that directly or indirectly increases academic engagement and decreases academic procrastination.

Methods: The present study was descriptive and of correlation and structural equations. The statistical population of the study included all non-profit high school male students in Hamadan city in the academic year 1398-99. Based on the two-stage cluster sampling method, 500 students were selected and completed Duckworth and Quinn's (2007) Grit scale, Reeve's (2013) academic engagement scale, Solomon \& Rothblum's (1984) academic procrastination, and Elliot, Murayama's, and Pekrun's (2011) achievement goal questionnaire. Data were analyzed using the structural equation statistical method and Spss24 and AMOS-26 software.

Results: The results showed that grit had a positive and significant effect on academic engagement with the mediating role of self-based and task-based development goals $(\beta=0.05, p<0.01)$. But grit did not have a negative and significant effect on academic procrastination with the mediating role of self-based and task-based achievement goals $(\beta=0.06, \mathrm{P}=0.68)$.

Conclusion: Self-based and task-based competencies can increase behavioral, cognitional, emotional, and agentic engagement in grittier students. But these competencies can't play a significant role in reducing procrastination.

* Corresponding Author: Javad Kavousian, Assistant Professor, Educational Psychology Department, Faculty of Psychology and Educational Sciences, Kharazmi University of Tehran, Iran.

E-mail: J_kavousian@yahoo.com

Tel: (+98) 9123585313

2476-5740/ (C) 2021 The Authors. This is an open access article under the CC BY-NC-ND license

(https://creativecommons.org/licenses/by-nc/4.0/). 


\section{Extended Abstract}

\section{Introduction}

Academic procrastination could be considered a serious threat to students' academic achievement and subjective well-being (Kim and Seo, 2015; Steel and Klingsieck, 2016) and it can lead to emotional discomfort and anxiety (Sénecal, Julien, \& Guay, 2003). Despite the findings of the negative consequences of procrastination, the role of students' individual differences in reducing academic procrastination has been less studied. According to previous researches, students' motivations are linked to individual differences in academic contexts (Burnette, O'Boyle, VanEpps, Pollack, \& Finkel, 2013; Chen \& Pajares, 2010; Robins \& Pals, 2002). Grit as an individual differences based construct, plays an important role in students' engagement, selfregulatory processes and success in challenging and demanding situations (Duckworth, Peterson, Matthews, \& Kelly, 2007; Duckworth \& Quinn, 2009; Eskreis-Winkler, Gross, \& Duckworth, 2018). Grittier individuals are thought to be powerful learners as they are less discouraged by failures and setbacks, more motivated to engage in strategic behaviors, and sustain a focused effort to achieve success (Eskreis-Winkler et al., 2018). They have a strong sense of agency. Thus, those individuals believe that they can achieve their goals and gain further expertise with persistent effort (Duckworth, 2016). In the other words, grit can increase insistence on the task during the challenges that the person poses for particular goals, and reduces the possibility of leaving the task ahead (Muenks, Wigfield, Yang \& O'Neal, 2017; Von Culin, Tsukayama \& Duckworth, 2014). Therefore, people are more likely to achieve their personal goals with higher levels of grit (Solmaz, Aksoy, Şengul, Sarışık, 2014; Wang \& Erdhiem, 2007; Von Culin et al., 2014). This can lead students to intrinsic motivation, increase academic engagement and reduce academic procrastination (Wolters, 2015; Robinson, 2015; Datu, 2016; Duchesne, Larose \& Bei, 2017). So far, no research has examined the personal goals of grittier students in increasing engagement and reducing their procrastination. Achievement goals as a forward- looking approach and an internal cognitive structure can engage the learner during academic assignments and cognitional, emotional, and behavioral regulation (Elliot \& McGregor, 2001; Senko, 2016). In the 3x2 model of achievement goals, on the basis of two competencies, the mastery goal was divided into selfbased and task-based achievement goals. But performance goal, did not change, and only was renamed as other-based goals (Elliot et al., 2011). In other words, in this model accuracy of the mastery goal has been increased.

According to this model, in task-based goal, the basis of evaluating competence is implicit in the task itself. So learners can receive feedback directly and immediately during task engagement. But the selfconcept is not conspicuous in such trying, as students' concentration remains task-focused. Because in selfbased goal, there is a more complicated situation and needs more cognitive potential than a task-based type (Elliot et al., 2011). Therefore, investigating selfbased and task-based goals can draw valuable attention to the learner and reduce concerns about the feedback of others. Because in other-based goals, learners receive competence information from another person, and the normative criterion of evaluation is not typically calibrated to give an optimal challenge (Nicholls, 1989). So in the present study, the other-based goals have not been examined. Thus grittier students can attempt towards achieving their long-term goals while persisting in the face of adversity (Duckworth, 2016), which in turn hinders the tendency to be procrastinated. Indeed, achieving challenging goals, such as better academic performance, requires the willingness to control impulses and work hard, as well as the ability to manage challenges associated with goal pursuit (Ivcevic and Brackett, 2014). This study is the first research that investigates the mediating role of taskbased and self-based achievement goals in the relationship between grit and both academic engagement and academic procrastination in high school students.

\section{Method}

Because of the coronavirus pandemic, there was a lack of access to all high schools in the city, thus nonprofit high school students participated in this study. 


\section{Monthly Journal of Psychological Science}

Vol. 20, No. 107, Winter(February) 2022
Furthermore considering male students is based on inconsistencies in previous researches results about gender differences in grit. Some researchers found gender differences in grit (Batres, 2011; Christensen \& Gerald 2014; Cross 2014; Stewart 2015). But the other researchers have found no gender differences (Batres 2011; Gorman 2015; Washington 2016). These inconsistencies suggest that gender-based differences in grit among students may be related to other variables but can change the results. Therefore the population of this study is all Iranian non-profit high school male students in Hamadan. two-stage cluster sampling was used, so in nine non-profit male high schools, two of them was selected randomly then all of the students in both of them (500 boys) participated and completed Duckworth and Quinn (2007) grit scale, Murayama and Pekrun (2011) achievement goal questionnaire, Solomon \& Rothblum (1984) academic procrastination scale and Reeve (2013) academic engagement. In order to data analyzing for structural equation modeling, Amos 26 was used.

Grit scale (Duckworth and Quinn, 2007) has 12 items that was created to assess perseverance in two subscales, consistency of interest (interest) and perseverance of effort (effort). 6 items for consistency of interest and 6 items for perseverance of effort. Responds are on a 5-point Likert scale of 1 (Very much like me) to 5 (Not like me at all). Higher scores in this scale is 60 and lower is 12. Cronbach's alpha coefficients for scale was 0.82 (Duckworth and Quinn, 2009). Within the current study Cronbach's alpha coefficient respectfully for interest and effort were 0.87 and 0.74 .

Achievement goal questionnaire (Murayama and Pekrun, 2011) has 18 items and 6 subscales: Taskapproach, task-avoidance, self-approach, selfavoidance, other-approach, other-avoidance goals, with 7-point Likert scale ranging from "strongly disagree" (1) to "strongly agree" (7). Cronbach's alpha coefficient was 0.84-0.93 (Elliot, Murayama and Pekrun, 2011). In different studies, factor analysis and validation on this questionnaire were done (David, 2012; Ning, 2018; Madigan, Stober and Pesfield, 2017; wang, Lio, Sang and cho, 2017). In this study, other-approach and other-avoidance goals weren't investigated to exploring the role of selfbased and task-based in students' goal setting. Within the current study Cronbach's alpha coefficient respectfully for Task-approach, task-avoidance, selfapproach, and self-avoidance goals were $0.84,0.85$, 0.86 and 0.87 .

Academic procrastination scale (Solomon \& Rothblum, 1984) have 27 items and three subscales: preparation for exams (8), doing the assignments (11) and preparing for the end of term paper (8). Questions number $7,8,18.26$ and 27 which were to assess two features of "discomfort about procrastination" and "willingness to change the habits of procrastination" were considered, And as the creator of the scale suggested, these six questions were not cited to be measured for the validity and reliability. Responds are on 5-point Likert scale of 1 (never) to 5 (always) and Cronbach's alpha coefficient for this scale was 0.75 (Rastegar et al, 2015). In this study preparing for the end of term paper subscale didn't investigate because teachers just used exams and assignments. Within the current study ronbach's alpha coefficient for both of preparation for exams and doing the assignments respectfully was 0.61 .

Academic engagement scale (Reeve, 2013) has a 17 items and 4 subscale scale that subscales are: cognitive (4 items), emotional (4 items), behavioral (4 items) and agentic engagements (5 items). Responds are on a 7-point Likert scale of 1 (completely agree) to 7 (completely disagree). Within the current study ronbach's alpha coefficient respectfully were $0.77,0.80,0.73$ and 0.85 and for academic engagements scale was 0.90 .

Table 1. Regression results (standardized regression coefficients) predicting the outcomes

\begin{tabular}{ccccc}
\hline Effect & $\beta$ & SE & C.R & P \\
\hline Grit to Academic engagement & 0.62 & 0.08 & 9.1 & Sig \\
Grit to Academic procrastination & -0.98 & 0.12 & -10.19 & Sig \\
Grit to Achievement goal & 0.22 & 0.07 & 3.26 & Sig \\
Achievement goal to Academic engagement & 0.32 & 0.07 & 4.47 & Sig \\
Achievement goal to Academic procrastination & -0.03 & 0.07 & -0.45 & Ns \\
***p $<.001$ & & & \\
\hline
\end{tabular}


Table 2. Bootstrap test results

\begin{tabular}{cccccc}
\hline Indirect Effect & $\beta$ & SE & sig & \begin{tabular}{c} 
Confidence interval \\
\cline { 4 - 6 } \\
Bounds
\end{tabular} & $\begin{array}{c}\text { Upper } \\
\text { Bounds }\end{array}$ \\
\hline Grit to Achievement goal to Academic engagement & 0.05 & 0.02 & 0.01 & 0.02 & 0.09 \\
Grit to Achievement goal to Academic procrastination & 0.06 & 0.01 & 0.68 & -0.02 & 0.02 \\
$\quad * * * p<.001$ & & & & & \\
\hline
\end{tabular}

\section{Results}

First, for the basic assumption of normality of observation, the Kolmogorov-Smirnov test was used. The results showed that the values of this test are significant for the research variables from 0.01 to 0.05 ( $\mathrm{p}<.001)$. But this test rejects the hypothesis of normality in a large sample size. For this reason, skewness and kurtosis of data were used.

The values for the skewness and kurtosis were less than 2 , and these indicate no significant difference with the normal distribution. Also, the normal Q-Q plot of the observed variables showed that the data points are close to the diagonal line. This result indicates the normality of the distribution of variables. In the second basic assumption of multicollinearity, variance inflation factor (VIF) and tolerance results showed that the tolerance values of the variables ( 0.9 to 0.97 ) are above 0.1 , and values of variance inflation factor (VIF) for the variables were less than 10, (between 1 and 1/1). Thus there is no multi-collinearity between the variables.

The first hypothesis results indicate the significant role of grit and achievement goals in predicting academic engagement (Table 4). According to the Bootstrap test results (Table 5), achievement goals play a mediating role in the relationship between grit and academic engagement. Because the lower and the upper bound of the confidence interval does not include zero.

Results of the second hypothesis indicate the significant role of grit in predicting academic procrastination directly (Table 4). But the direct effect and mediating role of achievement goals are insignificant (Table 4). Because according to the Bootstrap test, the lower and the upper bound of the confidence interval include zero (Table 5).

\section{Conclusion}

To date, no research has investigated the mediating role of achievement goals in the relationship between grit and academic engagement. Therefore, it is not possible to compare this finding with similar studies. But some previous studies have examined the relationship between grit and academic engagement (Bowman et al., 2015; Robinson, 2015; Holbein et al., 2016; Datu, 2016; Karlen et al., 2019). The other studies investigated the relationship between grit and achievement goals (Wang \& Erdhiem, 2007; Solmaz et al., 2014; Liem, 2016; Karlen et al., 2019). Furthermore, some researchers studied the relationship between achievement goals and academic engagement (Samareh \& Khezri Moghadam, 2015; Duchesne et al., 2017). The results of direct effects are consistent with previous researches, but grittier students have long-term goals that could increase their engagement. Because all their focus will be on starting and continuing the chosen paths to achieve personal goals and they will experience more motivation and optimism towards the results ahead (Perez, 2015). Therefore, grit increases a person's performance towards the goal and causes them to spend more time and effort to achieve their goals (Eskreis-Winkler et al., 2014).

Grittier students that focus on their self-based and task-based goals will experience more engagement. Because they get feedback from comparing their current, past, and future conclusions, and there is no competition with others.

Although previous research has documented the predictors and correlates of academic procrastination in students, comparatively little is known about the role of grit as a longer volitional processes of goal striving, on achievement goals; besides, the knowledge about direct and indirect effects of grit hinder academic procrastination, are still underexplored. In this study, consistent with previous research, grit predicted academic procrastination significantly (Wolters \& Hussain, 2014; Wolters, 2015; Siah et al., 2019). But task-based and self-based goals of students didn't motivate them to reduce their 
procrastination, and this direct effect was inconsistent with previous studies (Howell \& Watson, 2007; Howel \& Buro, 2009; Hashemi et al., 2015).

Given the gaps in the existing literature, the second goal of this study was to investigate the mediating role of achievement goals in the relationship between grit and academic procrastination. The result showed an insignificant mediating role of achievement goals in the relationship between grit and academic procrastination. This finding might reveal the role of classmates in a person's procrastination. So the student may have delayed tasks by comparing himself to others or being influenced by conformity. Because in adolescence, boys might be influenced by their peers more than younger age groups. Thus postponing educational assignments is not perceived as essentially negative.

Furthermore, we can point to the higher levels of expectations of grittier students, who, due to the nonchallenging nature of their homework, have tried to challenge their abilities in the shortest remaining time by postponing them.

\section{Ethical Considerations}

Compliance with ethical guidelines: The authors extracted this article from the $\mathrm{Ph}$.D. dissertation of the first author, which was approved 03.09.2019 in the Faculty of Psychology and Educational Sciences, Kharazmi University, Tehran. Ethical considerations like gaining the informed consent of the participants and the confidentiality of responses were considered in this research.

Funding: This study was conducted as a Ph.D. thesis with no financial support.

Authors' contribution: The first author was the senior author, the second and third were the supervisors, the fourth and fifth were the advisors.

Conflict of interest: the authors declare no conflict of interest for this study.

Acknowledgments: I would like to appreciate the supervisors, the advisors, the participants, and the non-profit high schools' staffs in Hamadan, Iran. 
زمينه: هرجند يُوهشهاى بيشين به بررسى آثار مستقيم ويزگى شخصيتى عزم در بيشيينى اهمال كارى و دركيرى دانش آموزان يرداخته

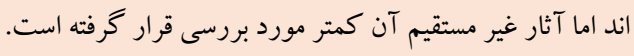

كليدواثهها:

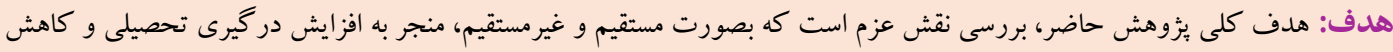

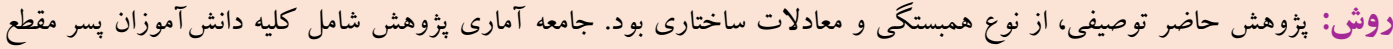

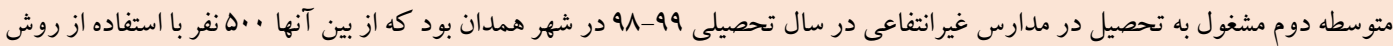

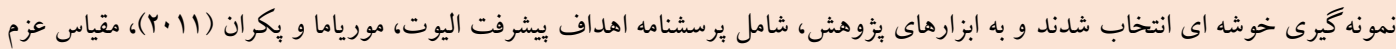

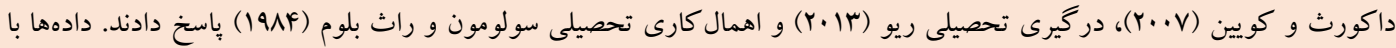

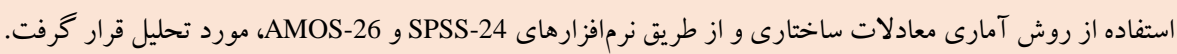

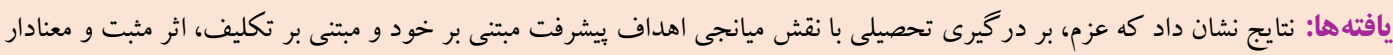

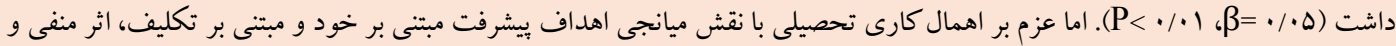

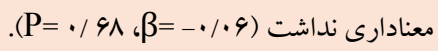

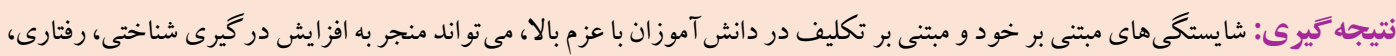


تلاش، بيشبينى كننده كليدى انخيزه، استراتزىهاى مديريت زمان و

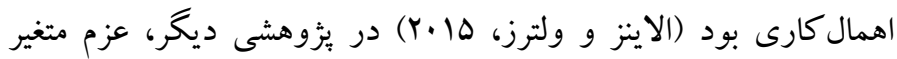

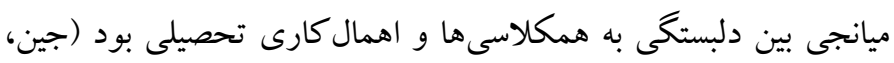

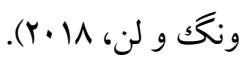
به نظر مىرسد، دانش آموزانى كه از عزم بالايى برخوردار هستند؛

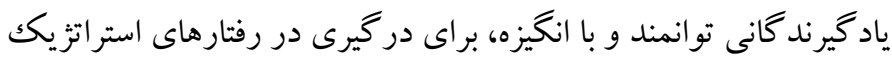
خو اهند بود كه شكستها و يسروىها، كمتر آنها را از اشتياق و تلاش

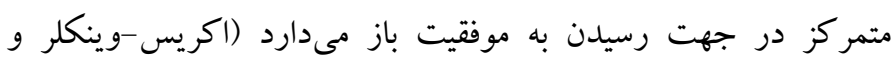

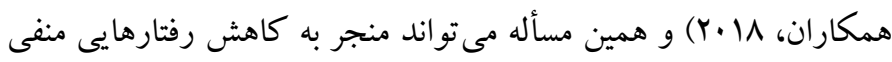

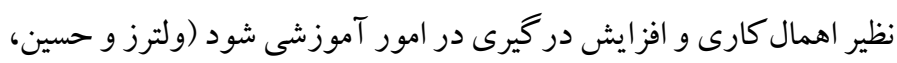

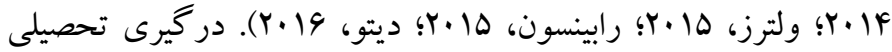

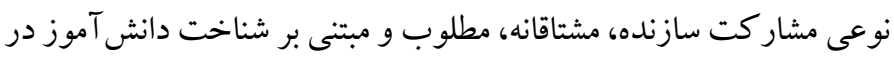

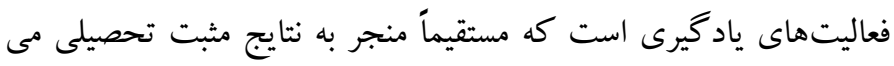

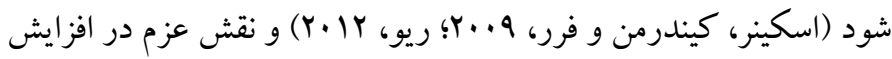

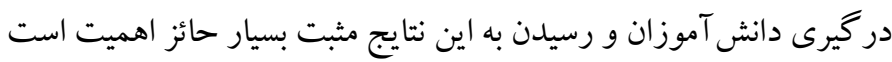
(هاج، رايت و بنت، IV. V). تاكنون هيج يثزوهشى به اين مسأله نيرداخته

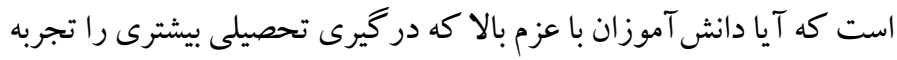

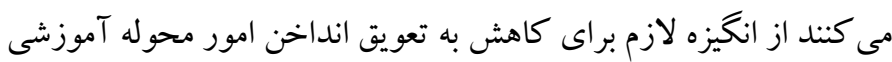

$$
\text { نيز، برخوردار هستند؟ }
$$

اشنايدر و همكاران (1991)، در نظريه اميد به دو عود عنصر مهم اشاره مى كنند

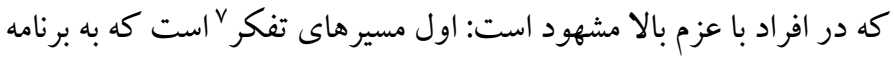
ريزى افراد براى دنبال كردن اهداف و نيز باورهاى شخصى مربوط است

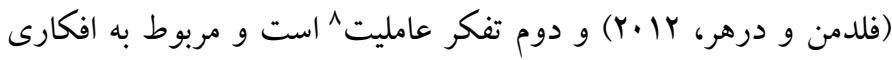

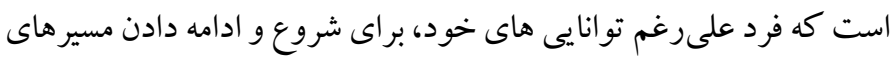

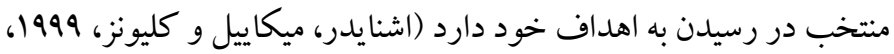

مقالمه

اهمال كارى تحصيلى را مى توان بعنوان خطرى براى موفقيت دانش آموزان

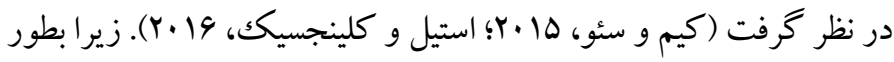

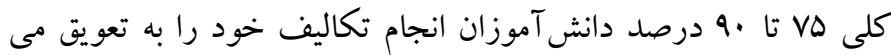

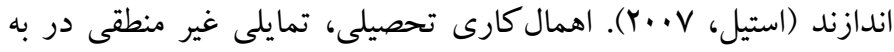
تأخير انداختن انجام تكاليف آموزشى است، تا حدى كه حتى باعث ايجاد

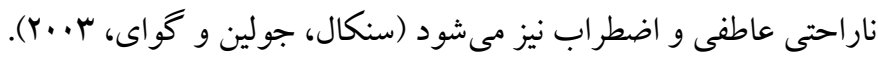

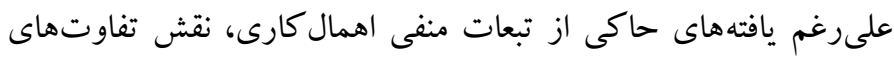

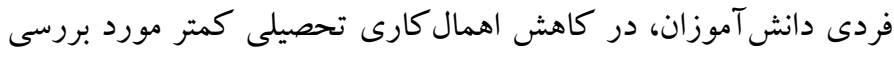

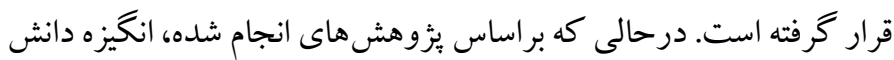
آموزان با تفاوتهاى فردى در محيطهاى آموزشى ارتباط دارد (برنت،

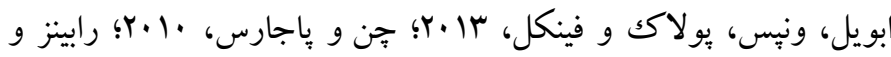

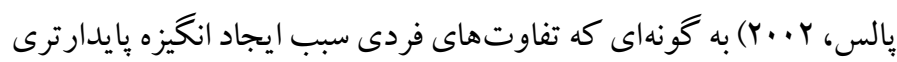

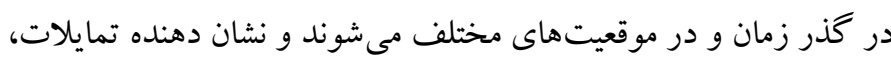
ارزشها، اهداف و ترجيحات بلند مدت به جاى خواسته ها و نيازهاى فردى

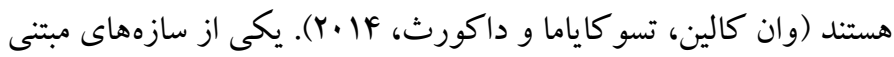

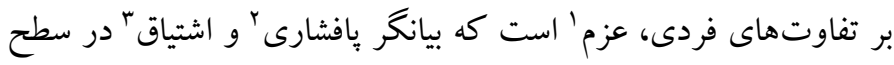

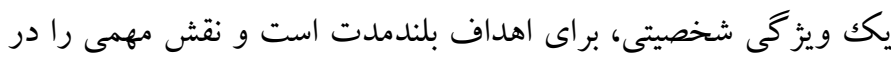

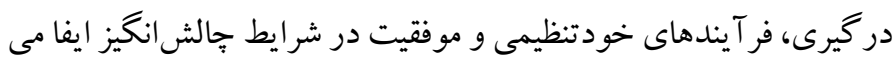

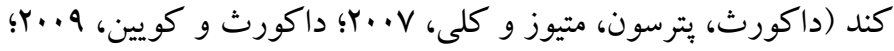

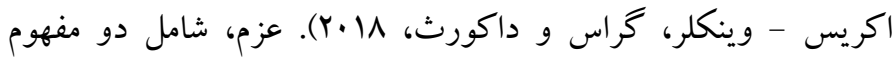
يافشارى در تلاش " و ثبات در علاقهه است به نحوى كه علاقه و تلاش

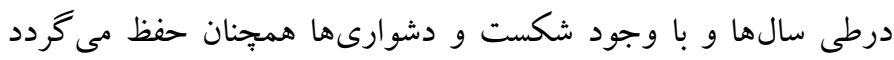

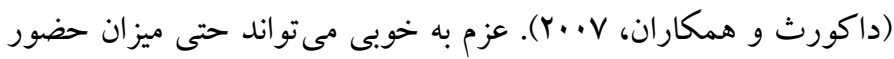

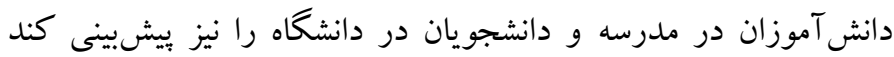

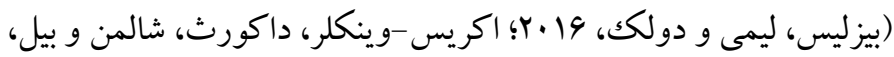

$(Y \cdot \mid Y$

طبق يُزوهش هاى انجام شده، ميز ان اهمال كارى در دانش آموزان با عزم بالا،

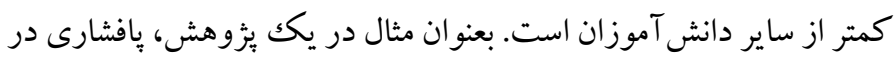

1. Grit

2 . perseverance

${ }^{3}$. passion

${ }^{4}$. perseverance of effort 


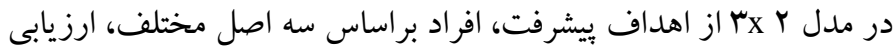
مىشوند: ا. شايستكى كنونى، كذشته و آينده خود، r. تكليفى كه شايستگى در آن بكار مىرود، آ. براساس عملكرد يا شايستخى ديكران (اليوت و همكاران، II (Y). با توجه به سه اصل مطرح شده و دو روش

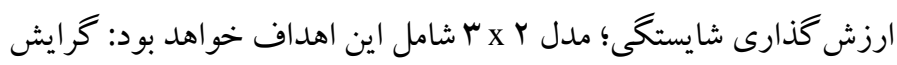
به تكليف Vا، مبتنى بر دست يابى به شايستكى در تكليف (مانند انجام درست تكليف)، اجتناب از تكليف؛، مبتنى براجتناب از عدم شايستكى در تكليف (مانند اجتناب از انجام تكليف نادرست)، كر ايش به خودو، مبتنى بر دست يابى به شايستكى درون فردى (مانند انجام تكليف بهتر از قبل)، اجتناب از خود ‘'، مبتنى بر اجتناب از شايستگى درون فردى (مانند اجتناب از بدتر انجام دادن تكليف نسبت به قبل)، گرايش به ديخران" بين فردى (مانند انجام تكاليف بهتر از ديكران) و اجتناب از ديكران"،

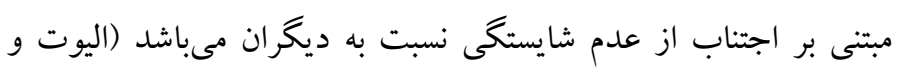

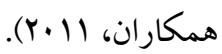
بطور كلى، مدل X Y به دنبال افزايش دقت درمورد هدف گذارى هاى تسلطى است تاحدى كه آنها را به دو دسته مبتنى بر تكليف و مبتنى بر خودفرد، تقسيم كرده است (اليوت و همكاران، ||r(Y). در معيارهايى كه مبتنى بر شايستكى درخود تكليف هستند؛ شخص مى تواند بازخورد را مستقيم، فورى و درطى دركيرى در انجام تكليف نيز دريافت كند اما استفاده از معيارهاى مبتنى بر خود، بيجيجيده تر است و نيازمند ظرفيت شناختى بيشترى است. حتى در سادهترين حالت آن، تنظيم مبتنى برخود"ا، نيازمند توانايى بازسازى شناختى دو خروجى بصورت همانند است (كه يكى از آنها مربوط به زمان حال است و ديخرى نيست) (اليوت و همكاران، II (Y). بنابراين، استفاده از اطلاعات مربوط به خود، بعنوان مركز خودتنظيمى، نقش (اخودا) را برجسته تر ساخته و مىتواند توجه ارزشمندى را به فرد

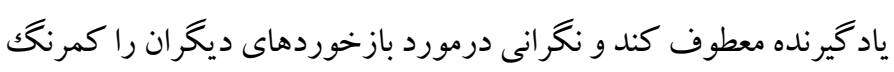

${ }^{8}$. task-avoidance

9. self-approach

${ }^{10}$. self-avoidance

${ }^{11}$. other-approach

12 . other-avoidance

${ }^{13}$. self-based regulation
دانش آموزانى كه از سطوح بالاترى از عزم برخوردار هستند؛ اين تفكر قدرتمند عامليت' را تجربه مى كنند. اين قبيل افراد، معتقد هستند كه با تلاش مداوم به تمام اهداف خود و تجربه و تخصص بيشترى دست خواهند يافت (داكورث، 19 +r). به عبارت ديخر، عزم مى تواند ميز ان بِافشارى در انجام تكليف را درطى جالث هاى موجود، براى رسيدن به اهداف خاص افزايش داده و احتمال تركك تكليف بيش رو را كاهش دهد (موئنكس، ويكفيلد،

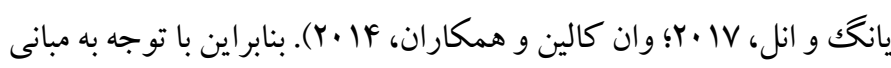
يثو هشى و نظرى، سطوح بالاتر عزم، احتمال دست يابى به اهداف شخصى

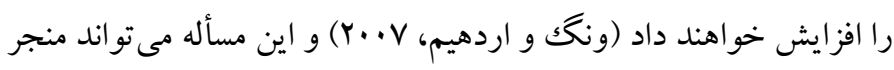
به ايجاد انگيزه در فرد شده و افزايش در گيرى تحصيلى و كاهش اهمال كارى تحصيلى را در بيى داشته باشد (ولترز، ها ·r؛ رابينسون، ها •ب؛ ديتو،

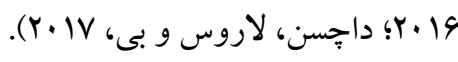
اما عزم به عنوان فر آيندى ارادى در هدف گذارى بلند مدت، هنوز به اندازه كافى در بين يزٔوهشكران شناخته شده نيست و با وجود اينكه اهداف بيشرفت به عنوان يكك رويكرد رو به آينده و يكك ساختار شناختى درونى، دركير شدن ياد گيرنده در جريان تكاليف تحصيلى و تنظيم شناختى، هيجانى و رفتارى را به عنوان يكك هدف دنبال مى كند (اليوت و مكك

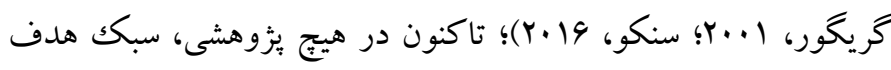
كذارى دانش آموزان برخوردار از سطوح بالاى عزم در افزايش در گيرى و كاهش اهمال كارى آنها، مورد بررسى قرار نخر فته است.

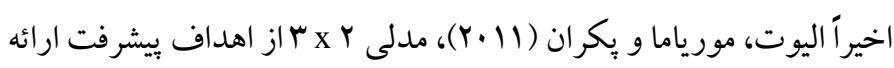
كردهاند كه در اين مدل هدف تسلطى ب كه به مهارت آموزى، تلاش براى توسعه يادگيرى و شايستكىها مى يرداخت، با توجه به شاخصهاى

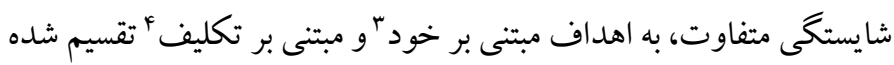
است، اما اهداف عملكردىه، تغييرى نكرده و فقط با نام جديد اهداف مبتنى بر ديخران 4، در مدل مذكور ارائه شده است.
1 . agency

2. mastery goal

${ }^{3}$. self-based

${ }^{4}$. task-based

5 . performance goals

${ }^{6}$. other-based goals

${ }^{7}$. task-approach 
دهند (داكورث، 19 · Y) كه به نوبه خود مانع از اهمال كارى مىشود. درواقع دست يابى به اهداف جالش انخيز مثل عملكرد تحصيلى بهتر، نيازمند تمايل

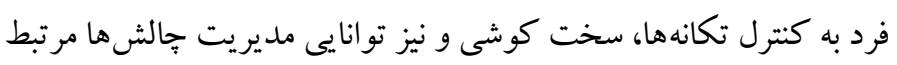

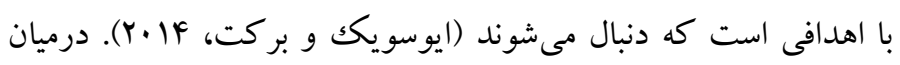

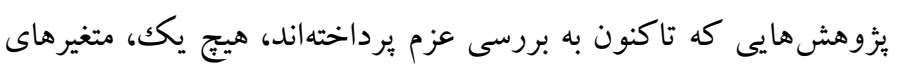

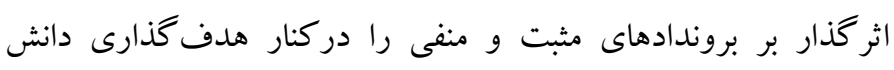

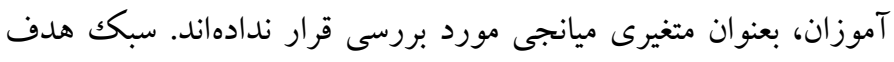

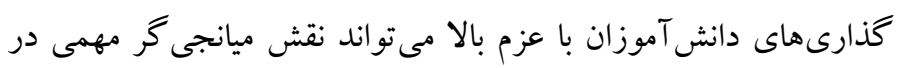

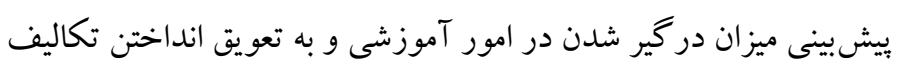

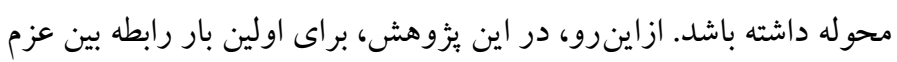
و دو متغير درگيرى تحصيلى و اهمال كارى تحصيلى با نقش ميانجى

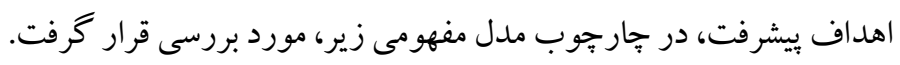

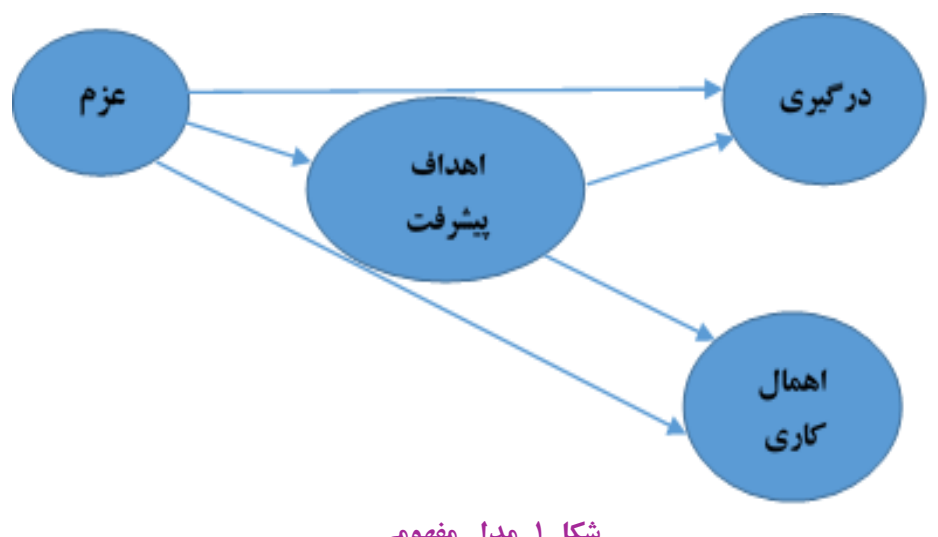

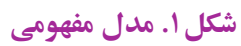

روش

الف) طرح ثخوهش و شر كت كنند كان: يُزوهش حاضر؛ از حيث هدف،

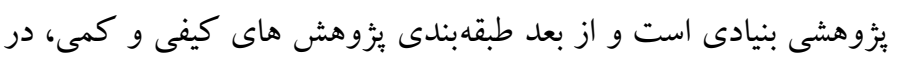

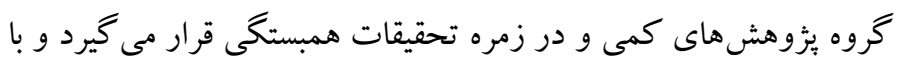

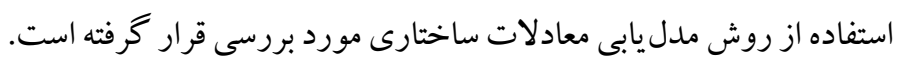

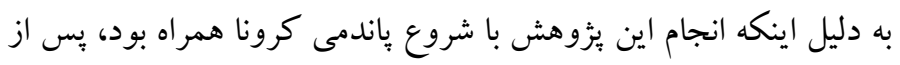

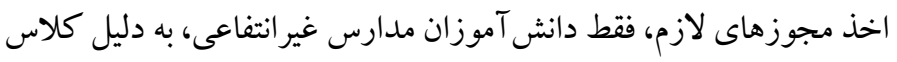
هاى فوقالعاده حضور داشتند كه با توجه به عدم دسترسى به ساير دانش

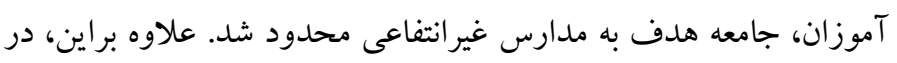

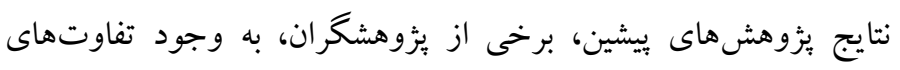

سازد، ازاينرو در بزوهش حاضر، اهداف گرايش به ديخران و اجتناب از ديخر ان مورد بررسى قرار نخر فتهاند.

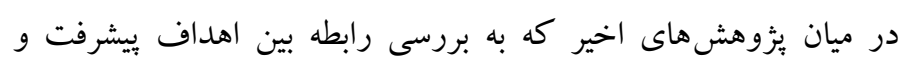

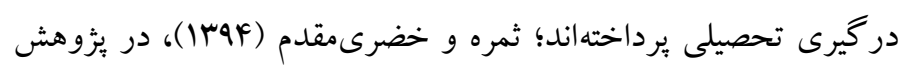

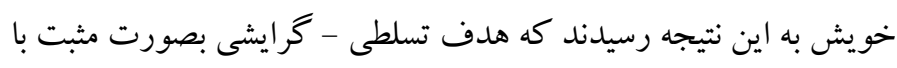

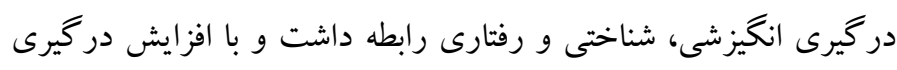

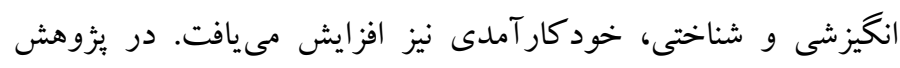

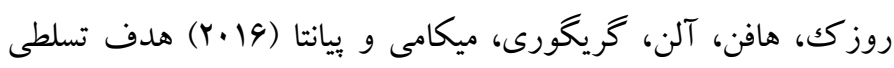

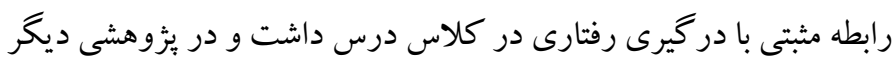
نيز، رابطهاى منفى، با عدم درگيرى در كلاس داشت (لئيم، لائو و نيه، (Y...人 در مورد رابطه بين اهداف بيشرفت و اهمال كارى تحصيلى نيز، برخى از يُزوهشهاى انجام شده، به اين نتيجه رسيدهاند كه هدف تسلطى با با اهمال

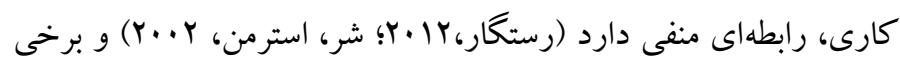
ديخر اذعان داشتهاند كه هدف تسلطى - كرايشى رابطهاى منفى با اهمال

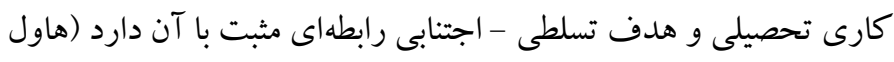

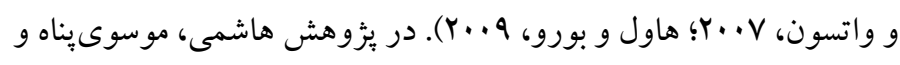

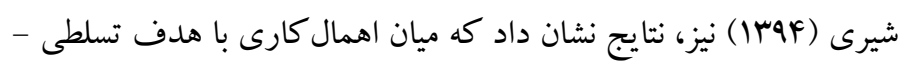

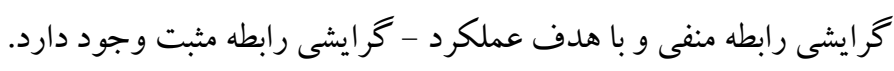

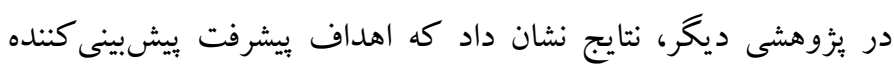

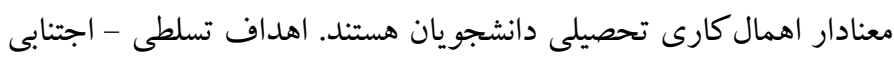

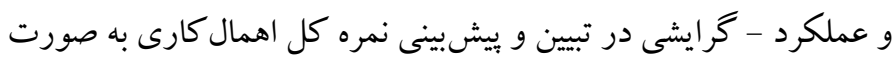

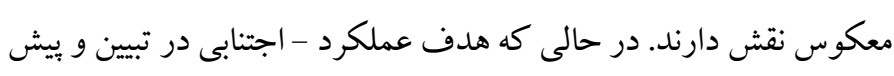
بينى نمره كل اهمال كارى به صورت مستقيم نقش دارد (حسينىمنش،

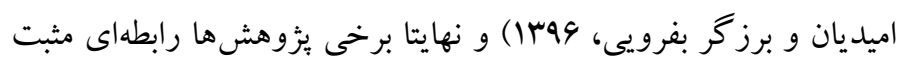

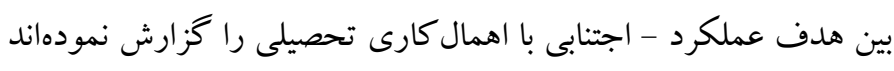

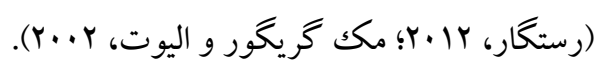

با توجه به يِينه و ادبيات يثوهش، عزم بعنوان يكك متغير مبتنى بر تفاوت

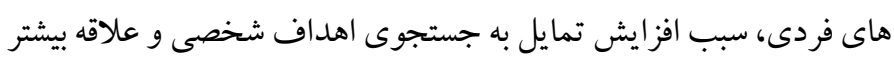
به تكاليف آموزشى و نهايتا منجر به اثرات مثبت در ياد خيرى مى مشود (وان

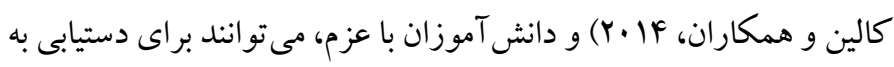
اهداف بلندمدت خود در مواجهه با دشوارىها، مداومت بيشترى به خرج 
و كويين (Y.9)، از تحليل عاملى و روش همسانى درونى استفاده كردند

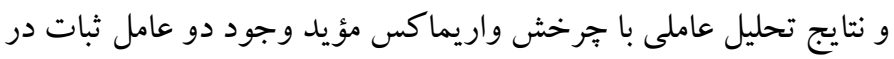

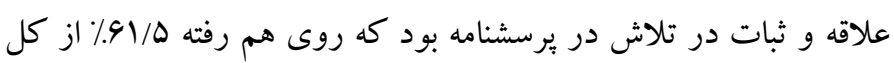

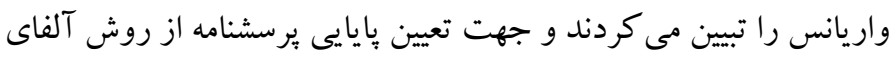
كرنباخ استفاده كردند و ضرايب حاصله براى ثبات عرد علاقه و ثبات در تلاش

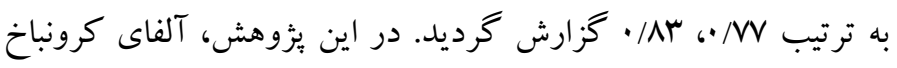
براى خرده مقياس هاى علاقه و تلاش، به ترتيب /AV · / • بدست آمد.

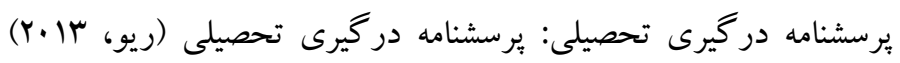

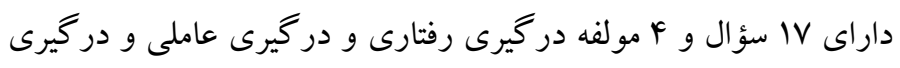

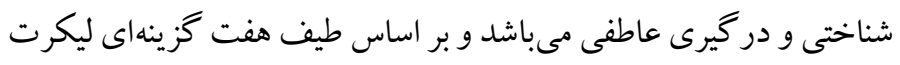

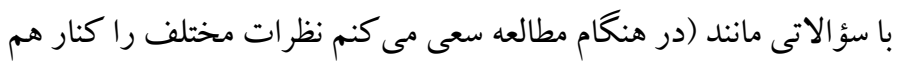

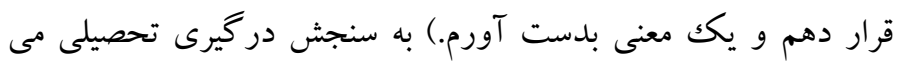
بردازد. هر سؤال داراى V امتياز مىباشد كه بسيار موافقم امتياز Vو و بسيار

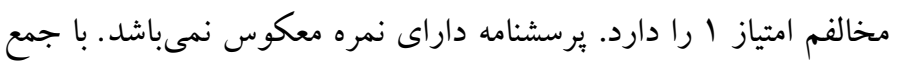

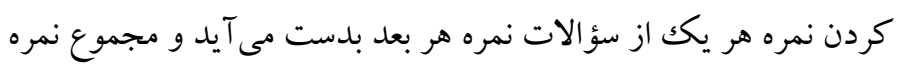

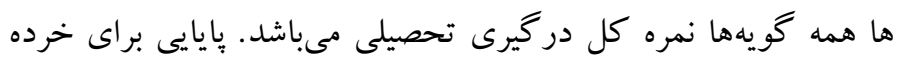

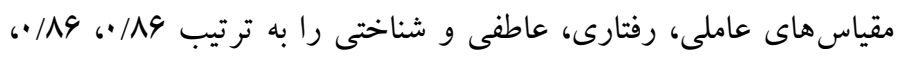

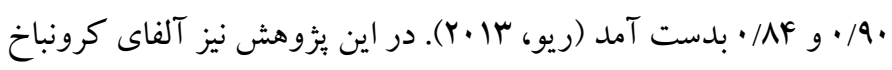

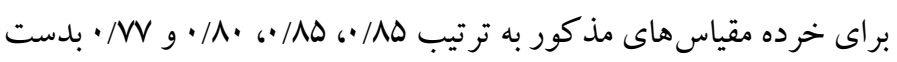

يرسشنامه اهداف ييشرفت: مقياس اهداف ويشرفت (اليوت و همكاران،

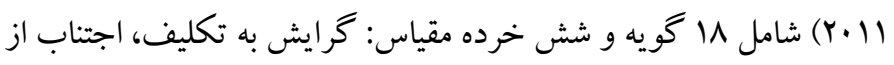

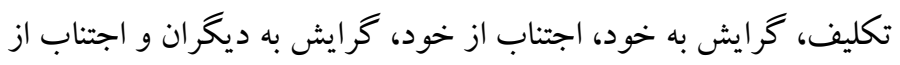
ديخران كه هر كدام از عاملها شامل سه گويه مىباشد، كه هر كر كدام از

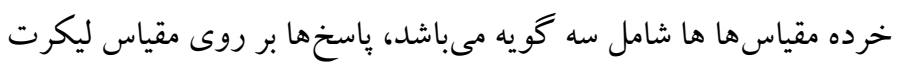

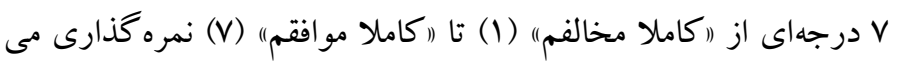

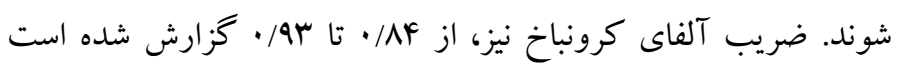

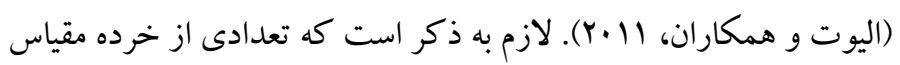

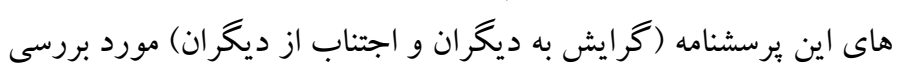

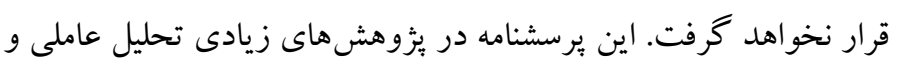

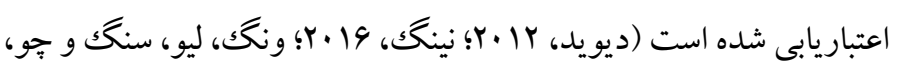

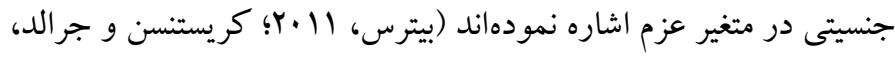

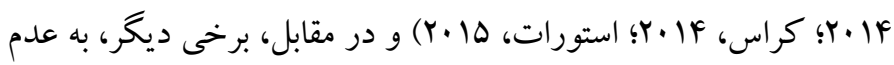

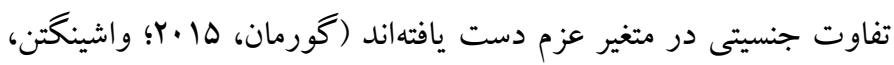

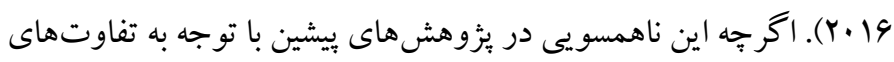
مبتنى بر جنسيت در عزم) مى تواند ناشى از ساير متغير هايى باشد كه در تغيير

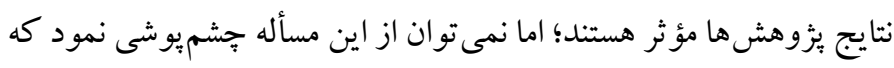

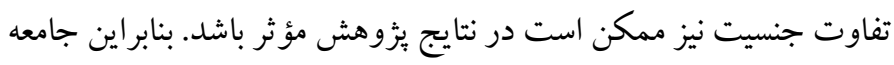

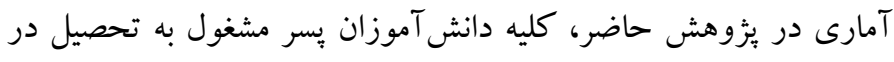

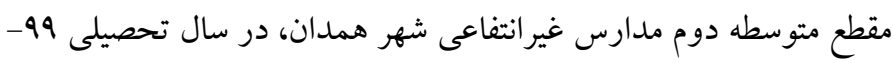

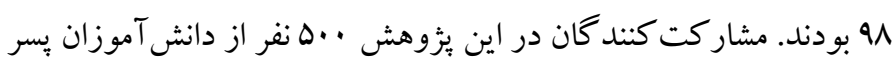

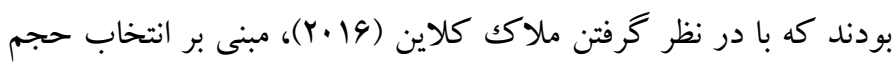

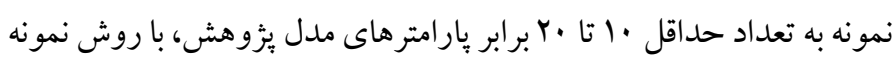

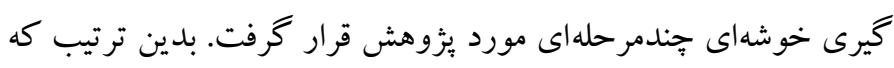

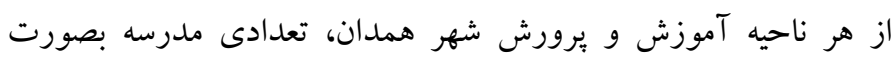

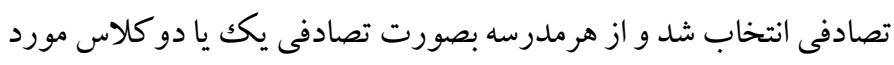

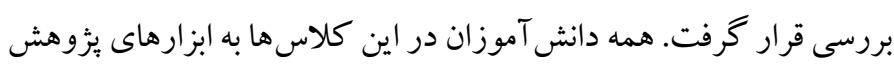
ياسخ دادند. ب) (بزار يرسشنامه عزم: براى بررسى ويزگى شخصيتى عزم از مقياس "عزم"

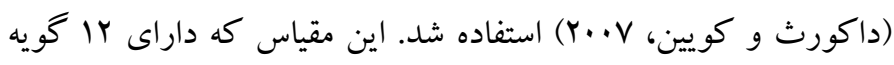

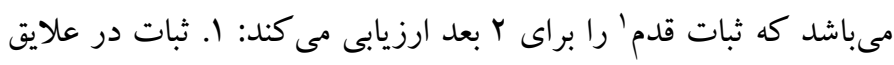

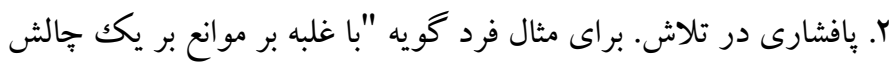

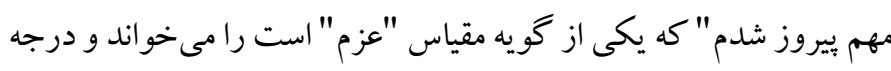

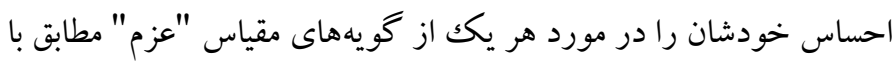

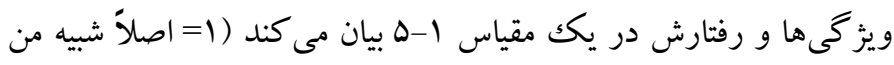

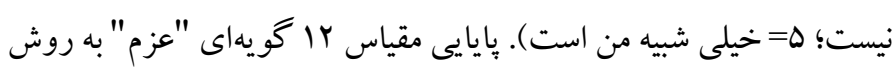

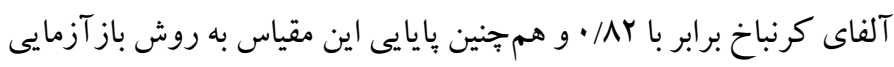

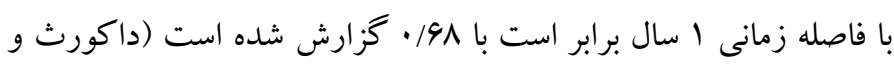

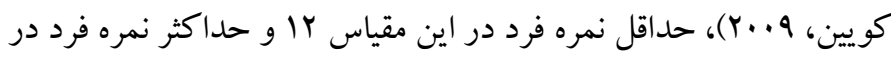

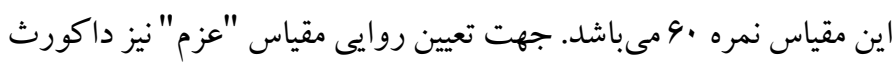

\section{Perseverance}


در روش تحليل عاملى، برابر M / • به دست آمده است (جو كار و

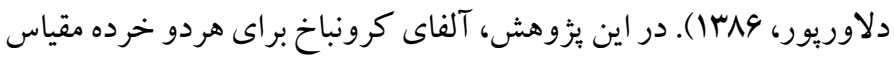
آمادگى بر اي امتحان و آمادكى براى تكليف، 191 • بدست آمد. جدول ا. ميانكين، انحر اف استاندارد، شاخصهاى كجى و كشيد

\begin{tabular}{|c|c|c|c|c|}
\hline كشيدگى & كجى & انحراف استاندارد & ميانگين & متغير \\
\hline \multicolumn{5}{|c|}{ 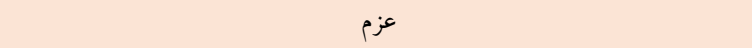 } \\
\hline$-\cdot / r$ & $\cdot / Y \wedge$ & $F / \Delta 9$ & $10 / 94$ & ثبات در علاقه \\
\hline$\cdot / r r$ & $-\cdot / 49$ & F/Tr & $r \cdot / r^{4}$ & يافشارى در تلاش \\
\hline \multicolumn{5}{|c|}{ اهمال كارى تحصيلى } \\
\hline - & .1 .9 & $F / V q$ & $19 / V Y$ & آمادگى امتحان \\
\hline$-\cdot / 4 \mid$ & $\cdot / \cdot v$ & $9 / \% \Lambda$ & $r r / \Delta$. & آمادگى تكليف \\
\hline \multicolumn{5}{|c|}{ اهداف يِشرفت } \\
\hline$-\cdot / 1$ & $-\cdot / \Delta \mathrm{A}$ & $r / r \Delta$ & $18 / 99$ & كرايش به تكليف \\
\hline . & $-\cdot / \wedge 9$ & $f / 1$ & 191.9 & اجتناب از تكليف \\
\hline $1 / \pi \Delta$ & $-1 / 19$ & $r / 9 \mu$ & $|V / F|$ & كرايش به خود \\
\hline$\cdot \pi$. & $-\cdot / \wedge$ & $F / I V$ & 191.9 & اجتناب از خود \\
\hline \multicolumn{5}{|c|}{ درگيرى تحصيلى } \\
\hline $1 / \cdot 1$ &.$- / 94$ & $F / V q$ & $r \mid / \Delta I$ & در گيرى رفتارى \\
\hline ו ו ו//י & $-\cdot / A r$ & G/M & rr/AY & درگيرى عاملى \\
\hline $1 / 11$ & $-\cdot / 99$ & F/GY & $r M / \Lambda$. & درگيرى شناختى \\
\hline 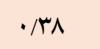 & $-\cdot /$ AS & $\Delta / r V$ & $r \cdot / q$. & درگيرى عاطفى \\
\hline
\end{tabular}

19 (Y). در اين بزوهش، آلفاى كرونباخ براى كرايش به تكليف، اجتناب

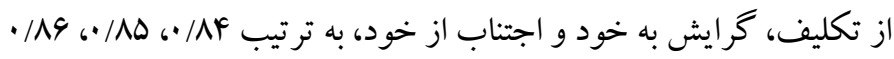

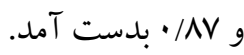
يرسشنامه اهمال كارى تحصيلى: يرسشنامه اهمال كارى (سولومون و

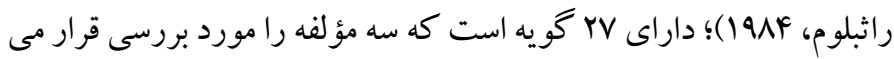
دهد. مؤلفه اول، آماده شدن براى امتحانات است كه ^ گويه را در بر مى كيرد. مؤلفه دوم، آماده شدن براى تكاليف است كه شامل ال كويه مى باشد و مؤلفه سوم، آماده شدن براى مقالههاى يايان ترم مىباشد كه شامل

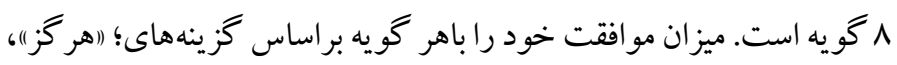
(به ندرت))، (أكهاهى)"، (اكثر اوقات)" و (هميشه)) مشخص مى كند. كه به

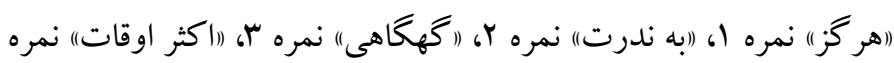
أ و (هميشه) نمره ها تعلق مى گيرد. در يثزوهش حاضر، آمادگى مقاله مورد بررسى قرار نمى گيرد. يايايى مقياس اهمال كارى تحصيلى از طريق همسانى درونى، در يثوهشى، ضريب آلفاى كرونباخ AF/ • به دست آمد (سولومون

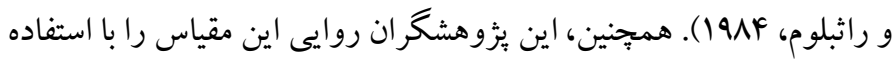
از همبستگى درونى، ^A / • بدست آوردند. در ايران و در بين جامعه آمارى دانشجويان يايايى اين مقياس با استفاده از ضريب آلفاى كرونباخ براى كل مقياس 19/ • و روايى آن با استفاده از آزمون كايرز - مير - اولكين (KMO)

\begin{tabular}{|c|c|c|c|c|c|c|c|c|c|c|c|}
\hline 11 & 1. & 9 & $\wedge$ & $\mathrm{v}$ & 4 & $\Delta$ & F & $r$ & $r$ & 1 & متغير \\
\hline \multicolumn{12}{|c|}{ 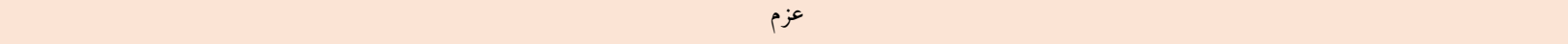 } \\
\hline & & & & & & & & & & & ثبات در علاقه \\
\hline & & & & & & & & & & $*_{*} \cdot / \uparrow$ & يافشارى در تلاش \\
\hline \multicolumn{12}{|c|}{ اهمال كارى تحصيلى } \\
\hline & & & & & & & & & $* *_{-} \cdot / 4 \wedge$ & $* * \cdot / 4 \Lambda$ & آمادكى امتحان \\
\hline & & & & & & & & $* * \cdot / \Delta 4$ & $* *_{-} \cdot / \mathscr{F V}$ & $* * \cdot / \Upsilon \wedge$ & آمادگى تكليف \\
\hline \multicolumn{12}{|c|}{ اهداف يِيشرفت } \\
\hline & & & & & & & $*_{-} \cdot / 19$ & $-\cdot / \cdot \wedge$ & $* * . / 19$ & $-\cdot / \cdot r$ & كرايش به تكليف \\
\hline & & & & & & $* * \cdot / v$ & $* *_{-} \cdot / 19$ & $*_{-} \cdot / \cdot 9$ & $* * . / 10$ & $-\cdot / \cdot 4$ & اجتناب از تكليف \\
\hline & & & & & $* * \cdot / \Delta 9$ & $* * . / F q$ & $* *_{-} \cdot / Y 4$ & $* *_{-} \cdot / r$ & $* * . / 19$ & $-\cdot / \cdot \Delta$ & كرايش به خود \\
\hline & & & & $* * . / 94$ & $* * \cdot / \Delta 9$ & $* * . / F \Delta$ & $* *_{-} \cdot / 10$ & $-\cdot / \cdot v$ & $* * \cdot / 10$ & $-\cdot / \cdot \Delta$ & اجتناب از خود \\
\hline \multicolumn{12}{|c|}{ درگيرى تحصيلى } \\
\hline & & & $* * \cdot / 19$ & $* * \cdot / Y \Lambda$ & $* * \cdot / r \mu$ & $* * . / 19$ & $* *_{-} \cdot / \uparrow r$ & $* *_{-} / \uparrow$ & $* * \cdot / \mu \Lambda$ & $*_{-\cdot / Y F}$ & درگيرى رفتارى \\
\hline & & $* * \cdot / \Delta \Delta$ & $* * \cdot / l f$ & $* * \cdot / r$ & $* * \cdot / \Lambda$ & $* * . / I f$ & $* *_{-} \cdot / r$ & $* *_{-} \cdot / r q$ & $* * \cdot / r q$ & $* *_{-} \cdot / Y I$ & درگيرى عاملى \\
\hline & $* * \cdot / F \Delta$ & $* * \cdot / \Delta F$ & $* * \cdot / r F$ & $* * \cdot / \pi$ & $* * \cdot / Y 1$ & $* * \cdot / r$ & $* *_{-} \cdot / \mu$ & $* *_{-} \cdot / \mu V$ & $* * \cdot / r \Delta$ & $* *_{-} \cdot /$ If & درگيرى شناختى \\
\hline$* * \cdot / \Delta \Delta$ & $* * \cdot / \uparrow \wedge$ & $* * \cdot / \Delta 4$ & $* * \cdot / r \mu$ & $* * \cdot / r r$ & $* * / r Y$ & $* * . / \Lambda$ & $* *_{-} \cdot / r$ & $* *_{-} \cdot / Y r$ & $* * \cdot / r \Lambda$ & $* *_{-} \cdot / 10$ & در گيرى عاطفى \\
\hline
\end{tabular}


جدول ب. شاخص هاى برازش

\begin{tabular}{|c|c|c|c|c|c|c|c|}
\hline RFI & IFI & RMSEA* & GFI & TLI & PCFI & $\chi^{2 / \mathrm{df}}$ & مدل \\
\hline.$/ 19$ &.$/ 94$ &.$/ . \Delta$ &.$/ 9$ &.$/ 91$ & .199 & $r / 9 Y$ & مدل نهايى \\
\hline
\end{tabular}

جدول ع. ضرايب استاندارد مسيرها

\begin{tabular}{|c|c|c|c|c|}
\hline $\mathrm{P}$ & C.R & SE & $\beta$ & 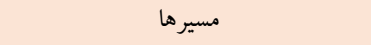 \\
\hline$* * *$ & $9 / 1$ & $\cdot / \cdot 1$ & .194 & عزم به درگيرى تحصيلى \\
\hline$* * *$ & $-1 \cdot / 19$ & $\cdot / l r$ &.$- / 91$ & عزم به اهمال كارى تحصيلى \\
\hline$* * *$ & $r / r q$ & $\cdot / \cdot \vee$ & $\cdot / r r$ & عزم به اهداف يِيشرفت \\
\hline$* * *$ & $F / F V$ & $\cdot / \cdot v$ & • & اهداف بيشرفت به درگيرى تحصيلى \\
\hline .194 & $-\cdot / \& \Delta$ & $\cdot / \cdot V$ & $-\cdot / \cdot r$ & اهداف ييشرفت به اهمال كارى تحصيلى \\
\hline
\end{tabular}

جدوله. نتايج آزمون بوت استراب

\begin{tabular}{|c|c|c|c|c|c|}
\hline \multicolumn{2}{|c|}{ فاصله اطمينان } & \multirow{2}{*}{ Sig } & \multirow{2}{*}{ SE } & \multirow{2}{*}{$\beta$} & \multirow{2}{*}{ 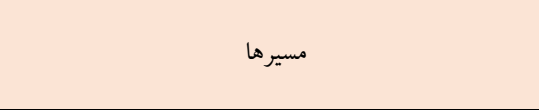 } \\
\hline حدبالا & حديايين & & & & \\
\hline.$/ \cdot 9$ & $\cdot \% r$ &.$/ 1$ & $\cdot / \cdot r$ & $\cdot / \cdot \Delta$ & عزم به درگيرى تحصيلى با ميانجى گرى اهداف يِيشرفت \\
\hline$\cdot / \cdot r$ & $-\cdot / \cdot r$ & .191 &.$/ \cdot 1$ & -.1 .4 & عزم به اهمال كارى تحصيلى با ميانجى گرى اهداف بيشرفت \\
\hline
\end{tabular}

بدست آمده براى متغيرها (بين ا تا (1/1) و كوجٍكتر از · ا هستند كه بيانكر عدم هم خطى جند گانه بين متغير هاست. در جدول ا، ميانگين، انحر اف استاندارد و مقادير كجى و كشيد كئى متغيرها

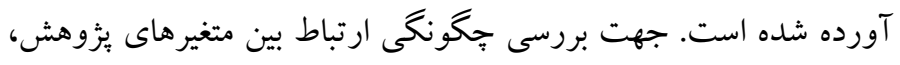

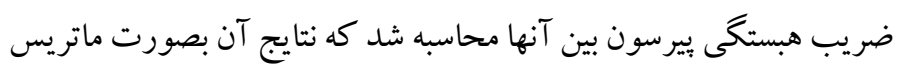
همبستكى در جدول r نشان داده شده است. با توجه به جدول r، ميزان همبستخى بين اكثر متغيرهاى يزوهش در در سطح

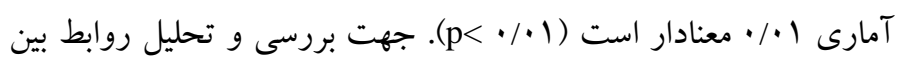

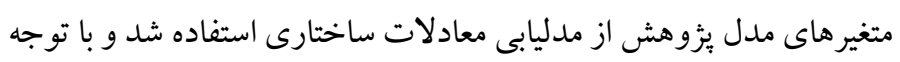

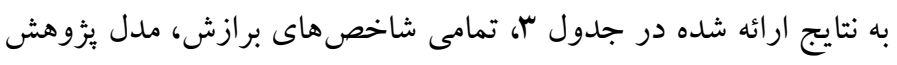

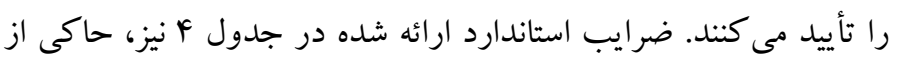
معنادار بودن تمام مسيرهاى مستقيم در مدل بِزوهش است و تنها مسير

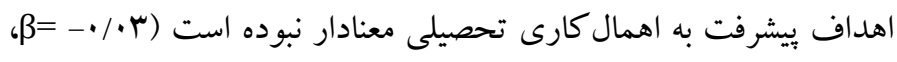

.$(P=\cdot / 94$

جهت بررسى فرضيه هاى بزوهش مبنى بر نقش ميانجى گر اهداف بيشرفت در رابطه بين عزم و در گيرى تحصيلى و همجنين در رابطه بين عزم و اهمال

${ }^{3}$. normal Q-Q plot
يافته ها

ابتدا براى اطمينان از اينكه دادههاى يُزوهش، مفروضههاى زيربنايى نرمال

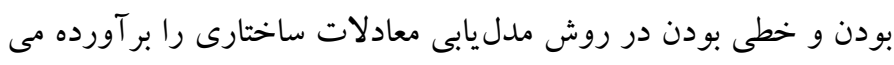

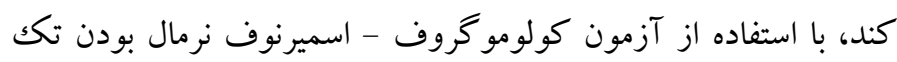

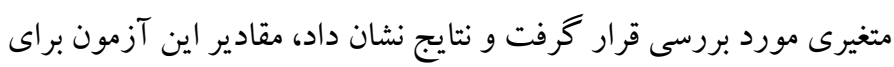

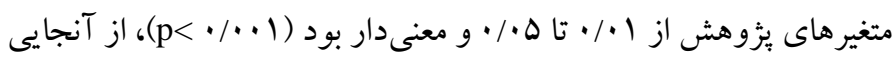

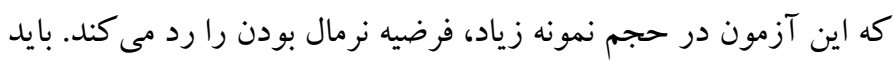

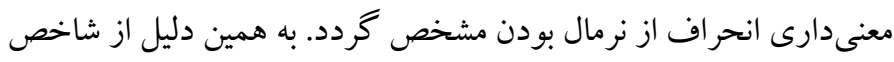

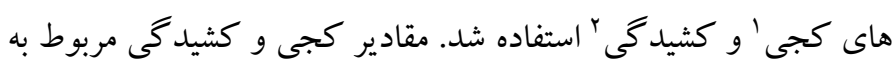

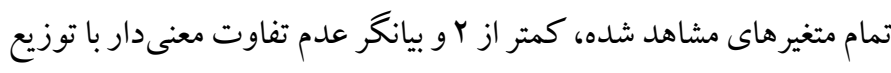

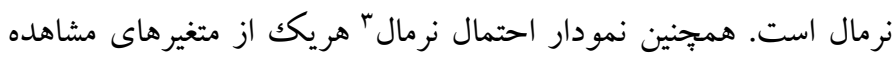

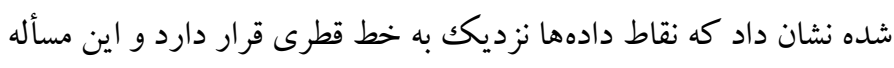

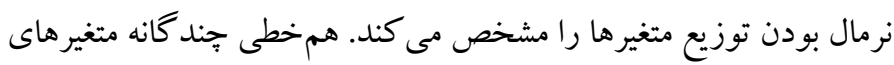

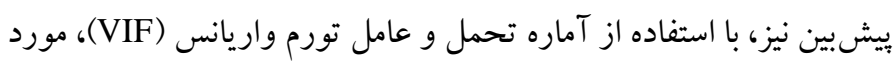

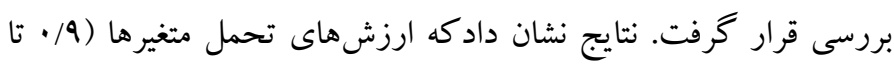

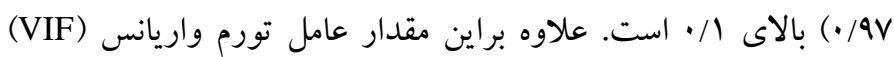

${ }^{1}$. skewness

${ }^{2}$. kurtosis 


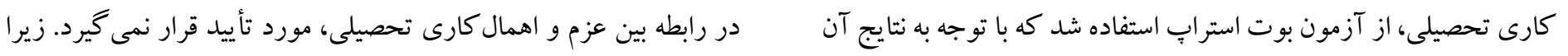

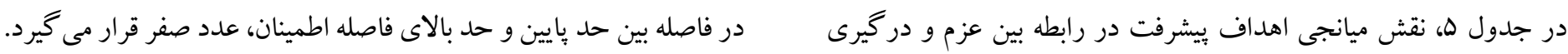

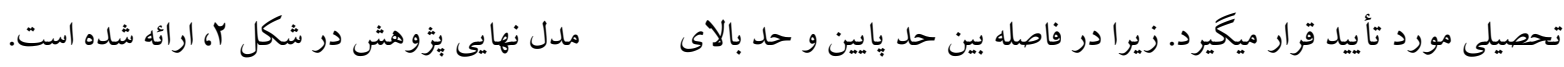

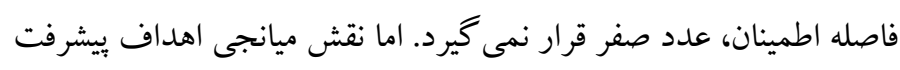

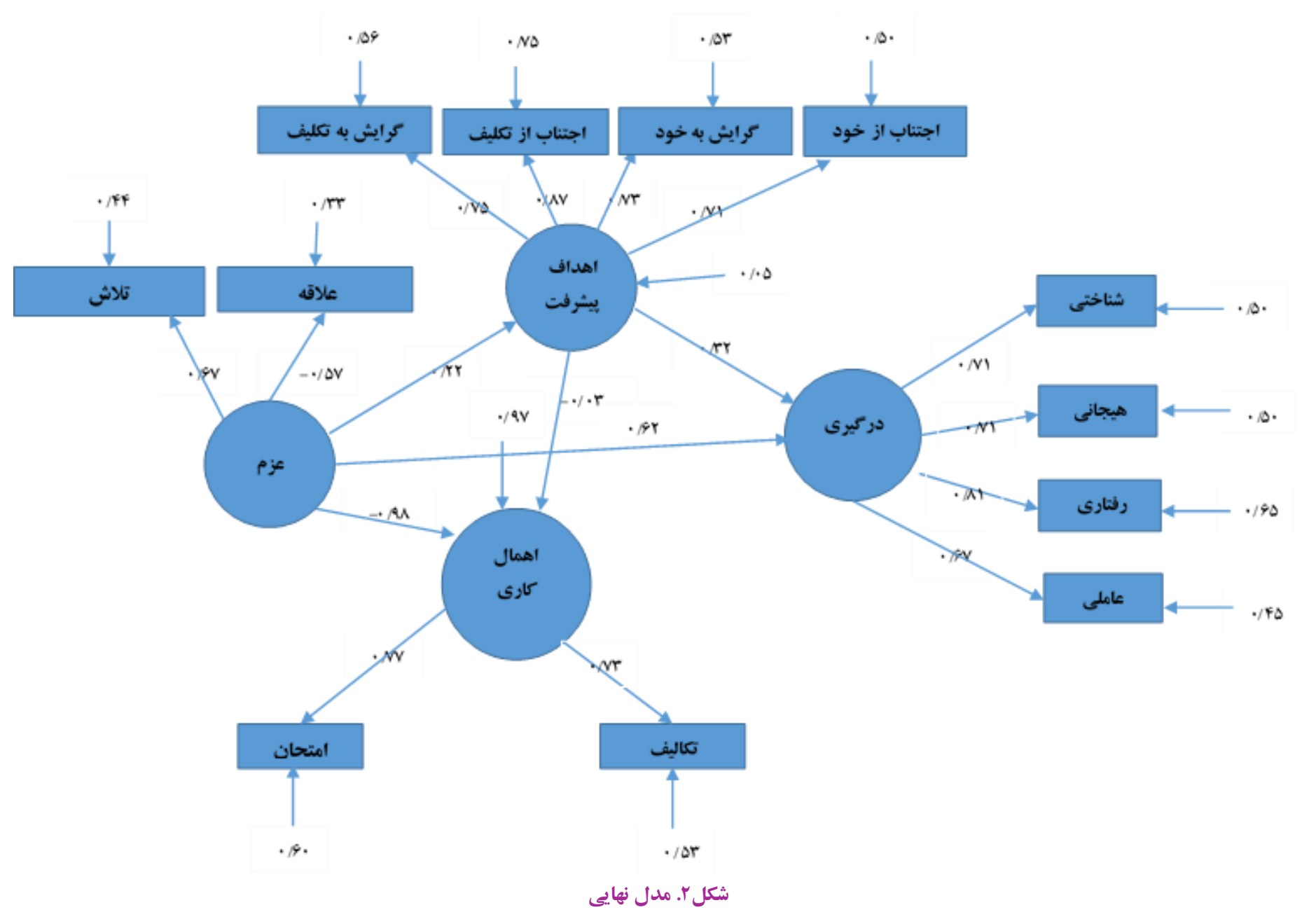

و در گيرى تحصيلى برداختهاند و رابطه مثبت و معنادار بين عزم و درگيرى

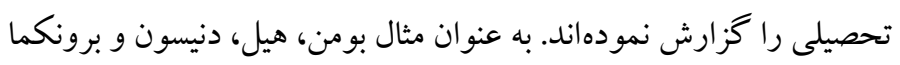

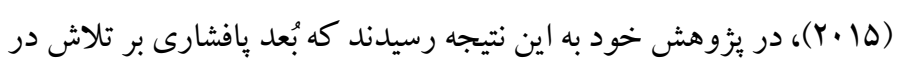
عزم، رابطه مثتى با در گيرى دانشجويان با دانشكده و همكلاسى ها داشت.

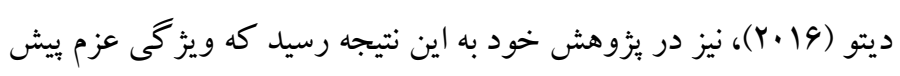

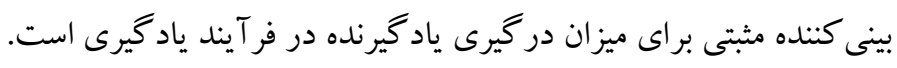

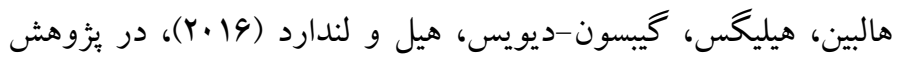
خود به اين نتيجه رسيدند كه دانش آموزان با عزم بالا، در آزمونهاى هيدي
بحث و نتيجه كيرى هدف اين يزوهش بررسى رابطه بين عزم با درگيرى تحصيلى و اهمال

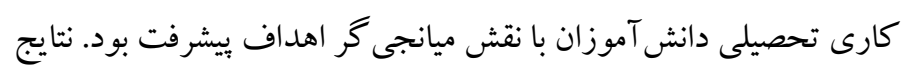
نشان داد كه نقش ميانجى گر اهداف بيشرفت در رابطه بين عزم و در خيرى دئي تحصيلى معنادار است. از آنجايى كه تاكنون بثزوهشى به بررسى هدف

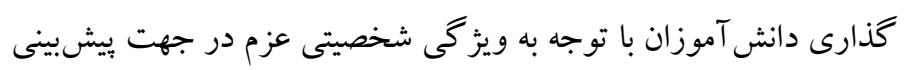

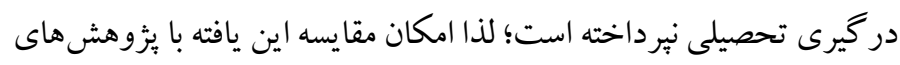

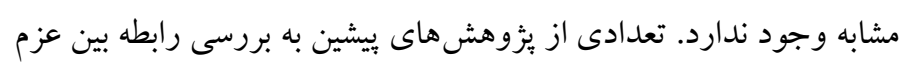


هاى هدف گذارى دانش آموزان برخوردار از سطوح بالاى عزم را در ميزان در گيرى تحصيلى آنها مشاهده نمود.

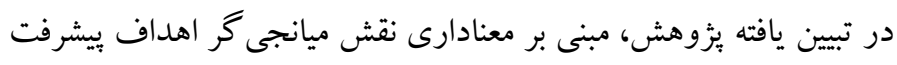

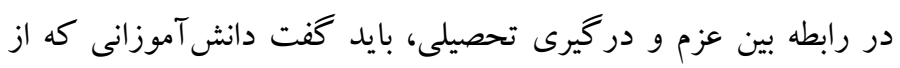

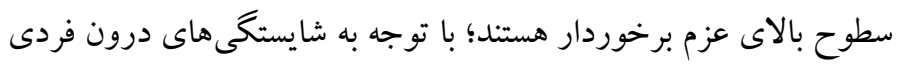
و مر تبط با تكاليف، هدف گذذارى كرده و عامدانه و فعالانه تلاش مى كنيند، مطالب مورد نظر براى يادگيرى را شخصىسازى و غنىسازى كنند تا

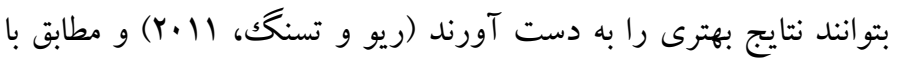
نظريه اميد اشنايدر و همكاران (1991)، تمام تمركز آنها بر شروع و و ادامه ورنه دادن مسيرهاى منتخب در رسيدن به اهداف شخصى به كار گرفته خو اهد

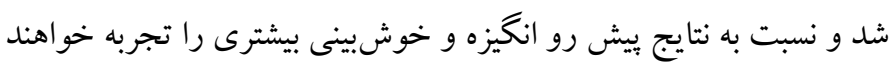

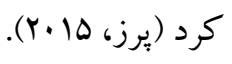

بنابر اين عزم نه تنها سبب افزايش عملكرد فرد نسبت به هدف مى شودد، بلكه موجب مى شود زمان و تلاش بيشترى را براى دست يافتن به اهداف خوده،

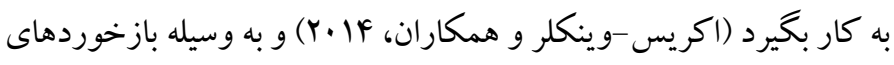
ناشى از هدف كذارى مربوط به عملكرد خويش در تكاليف و بروندادهاى شخصى، به رقابت با عملكرد بيشين به جاى رقابت با همكلاسى ها، بيردازد.

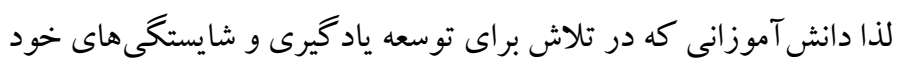

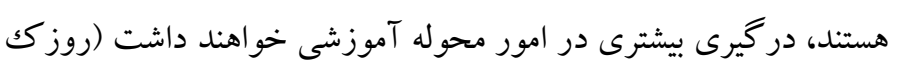

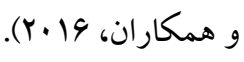
در مورد يافته ديخرى از يثوهش حاضر، نتايج نشان داد اهداف بيشرفت، وران، در رابطه بين عزم و اهمال كارى تحصيلى نقش ميانجى

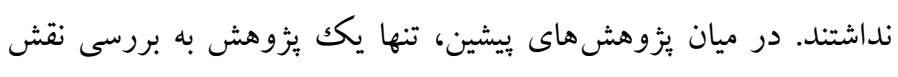

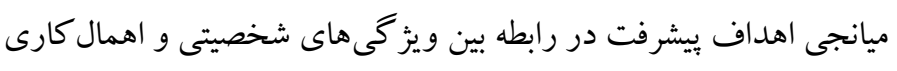

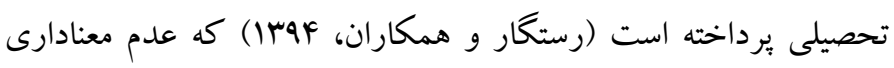

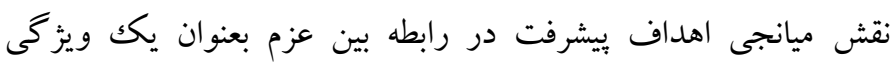

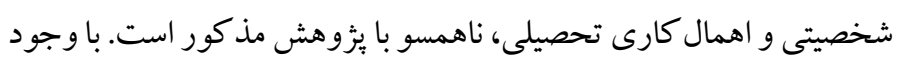

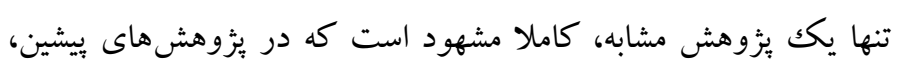
بررسى نقش هدف گذذارى دانش آموزان برخوردار از سطوح بالاى عزم در

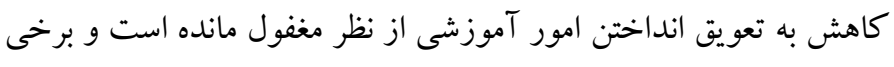

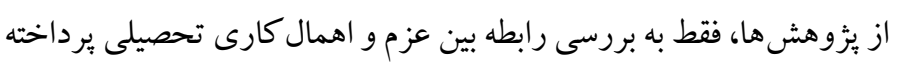

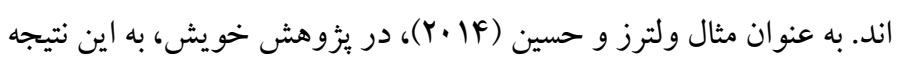

استاندارد بهتر عمل كردند و باورهاى قدر تمندى در مورد درگيرى نسبت

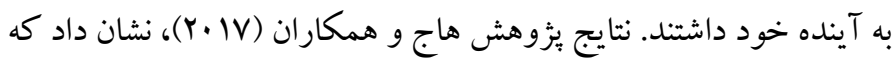

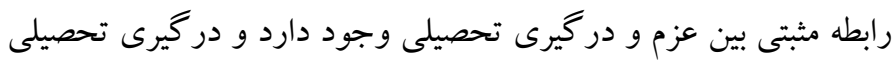
نقش ميانجى گر را در بين عزم و توليد كننده بودن تحصيلى ايفا مى كرد.

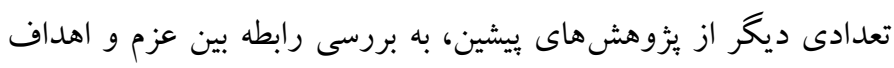

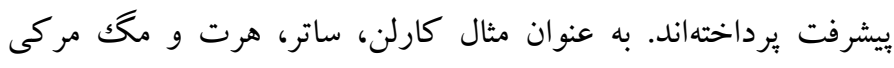

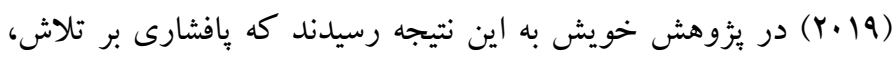

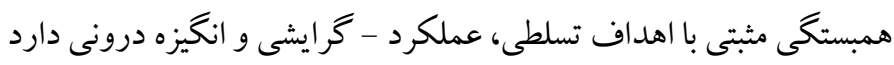

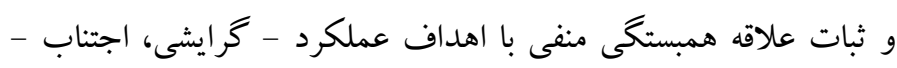

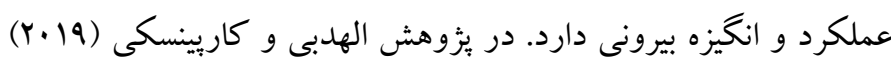

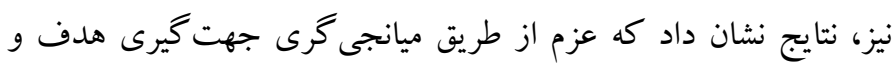
خو دكار آمدى تحصيلى، رابطه مثبتى با عملكرد تحصيلى دارد.

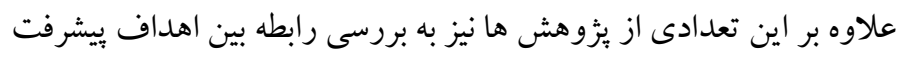

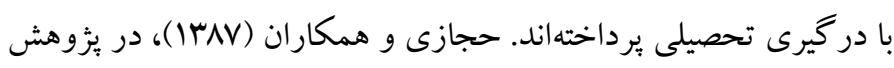

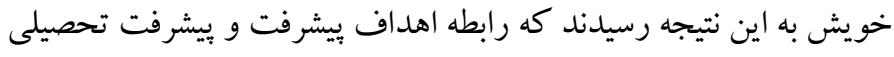

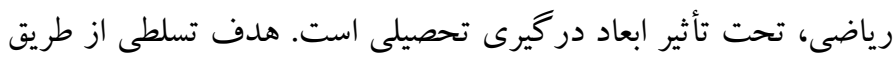

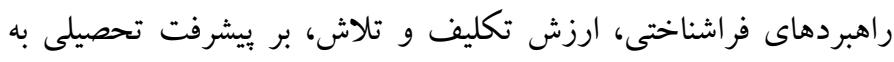

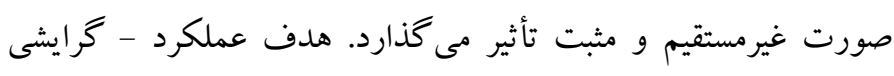

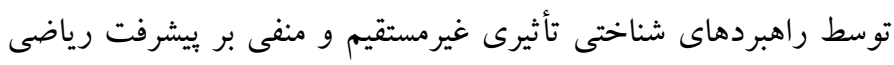

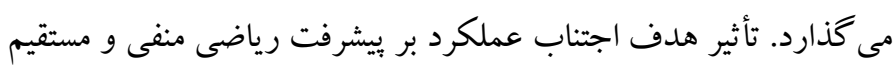
است. همجنين نقش واسطهاى ابعاد درگيرى تحصيلى در رابطه ميان

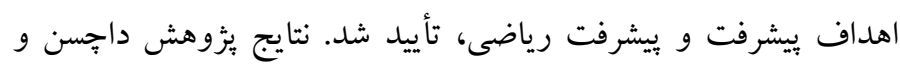

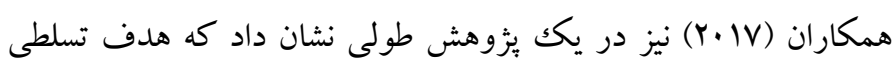

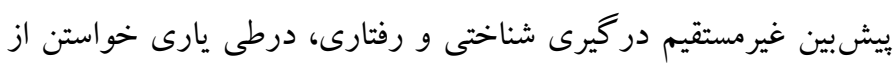

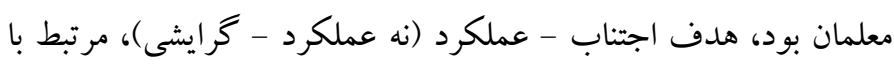
در گيرى شناختى در طى رفتار هاى يارى خواستن بودند.

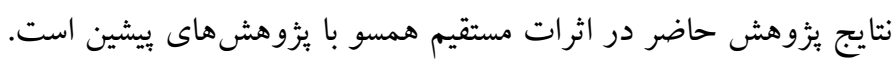

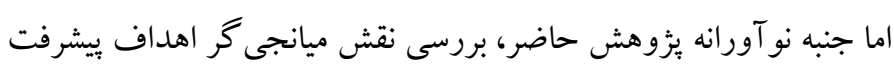

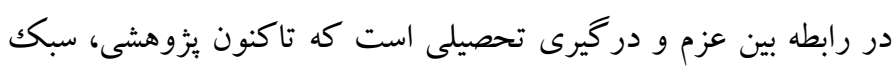

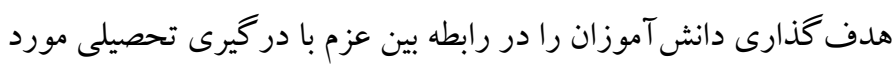

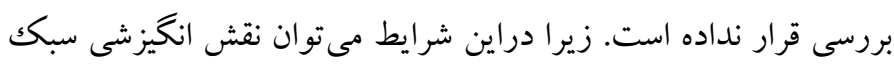




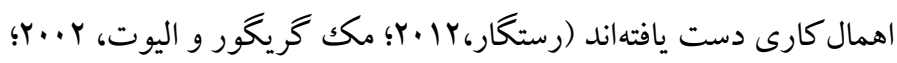

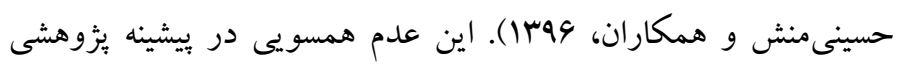
بيانكر نقش متفاوت ديخر ان در هدف گذارى هاست. جنسيت و دوره سنى

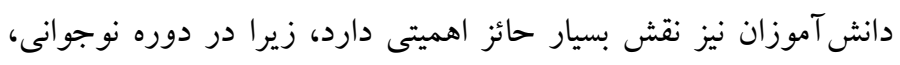

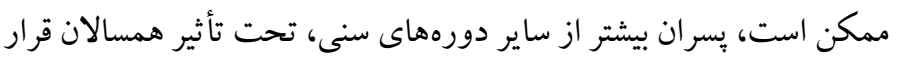

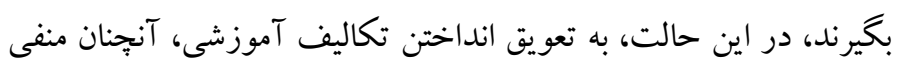
ادراكك نمى شود.

علاوه بر عدم همسويى در يزوهشهاى ويشين، جنسيت و نقش دوره

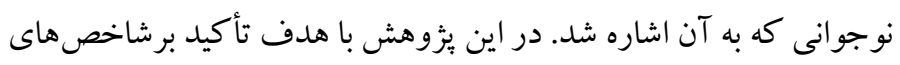

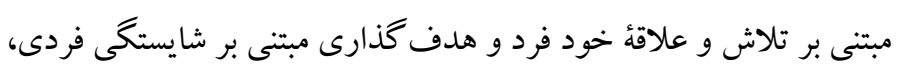

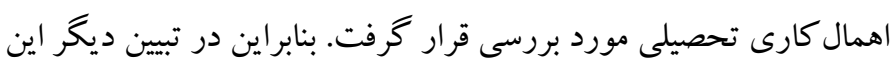

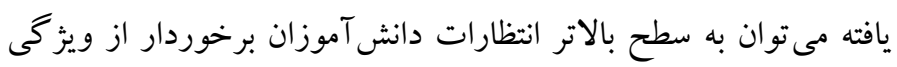

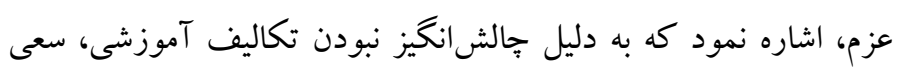

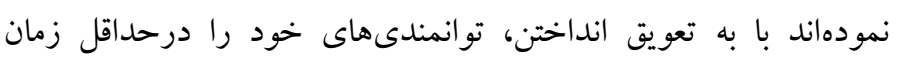

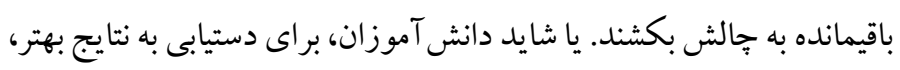

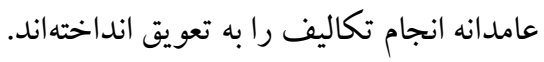

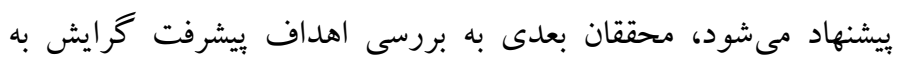

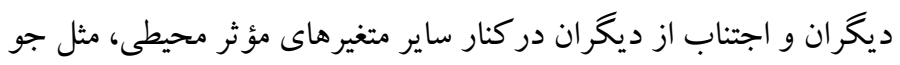

$$
\text { كلاس درس بيردازند. }
$$

اكرجه در نتايج اين مطالعه مى توان محدوديت هاى زيادى راذئن اذكر كرد. در ابتدا بايد مدنظر داشت كه مدل تحقيق، مبتنى بر تحقيقات قبلى و بيشينه

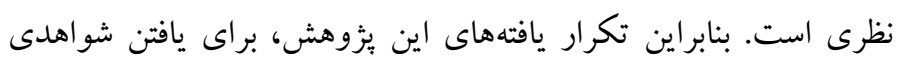

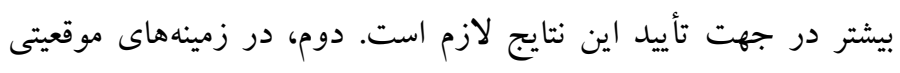

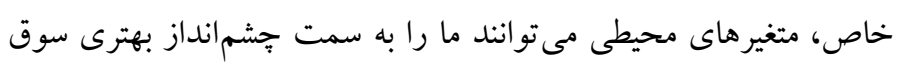
دهند. زيرا در اين يزوهش، اهداف بيشرفت حتى اهمال كارى تحصيلى را مار

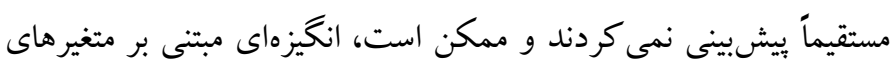

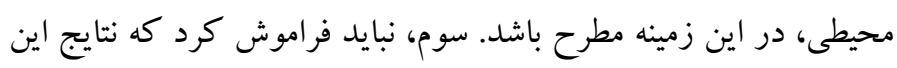
مطالعه ممكن است به دليل جنسيت شر كت كنند كان با محدوديت مو اجنه

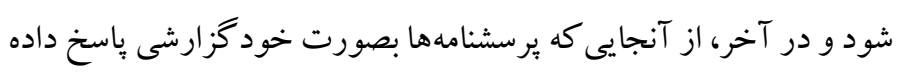

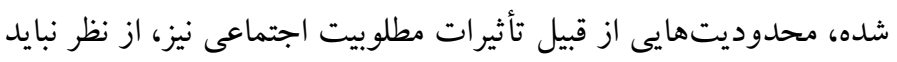
مغفول بماند.
رسيدند كه بين عزم و اهمال كارى تحصيلى، رابطهاى منفى وجود دارد.

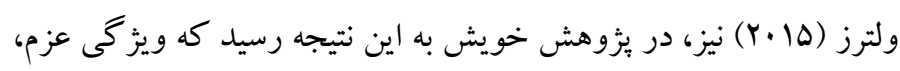

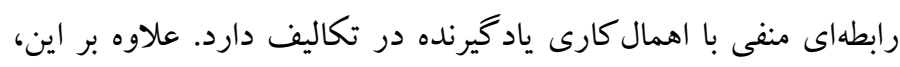

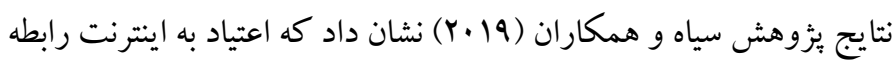

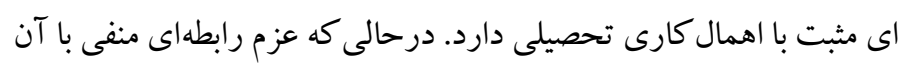

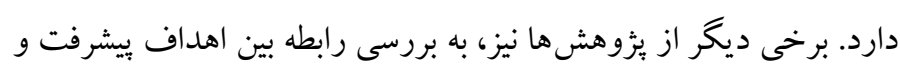

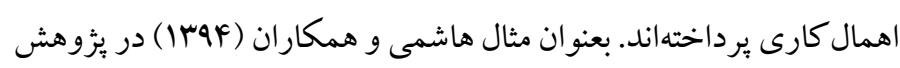
خويش به اين نتيجه رسيدند كه هدف تسلط - گر ايشى با اهمال كارى رابطه

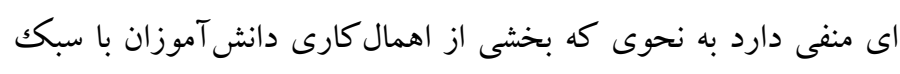

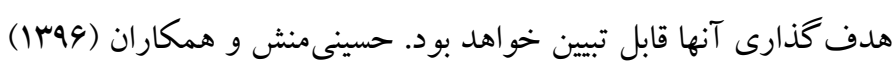

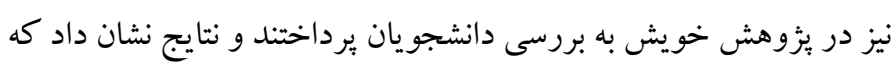
اهداف بيشرفت، بيشبينى كننده هاى معنادار اهمال كارى تحصيلى بودند.

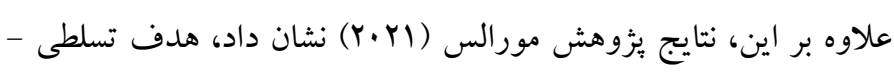

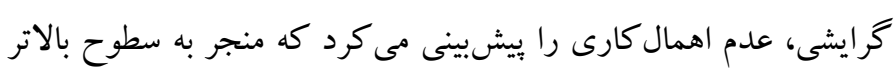

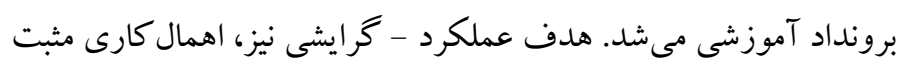

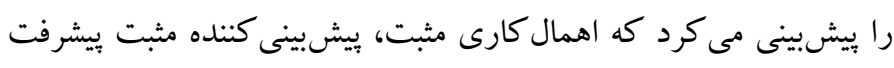

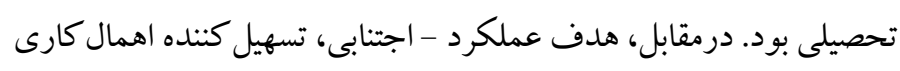
منفى بود كه منجر به بيشرفت تحصيلى ضعيف مى شد.

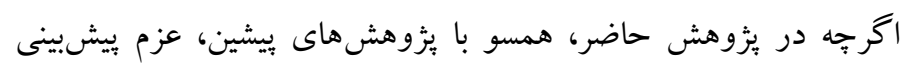

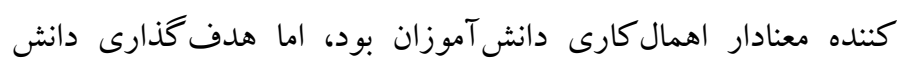

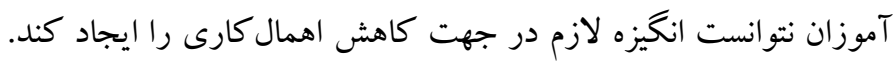

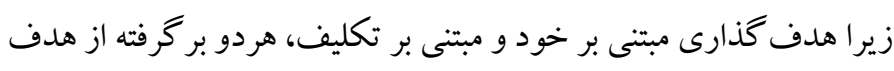

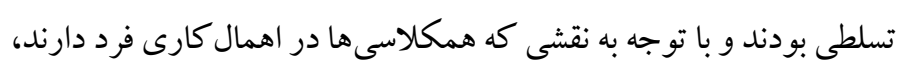

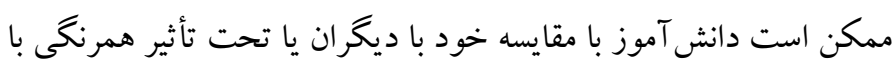
ديخر همكلاسىها، انجام تكاليف خود را به تعويق انداخته باشد. البته در

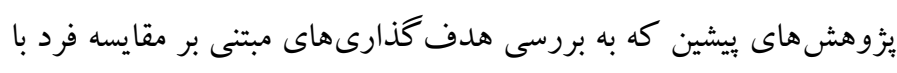

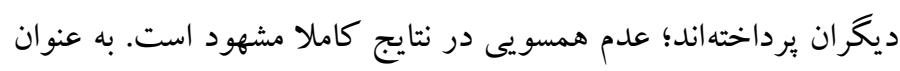

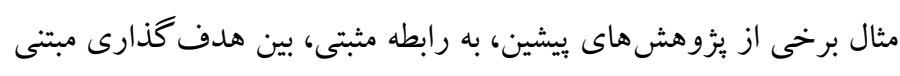
بر برترىجويى نسبت به ديخران (اهداف عملكرد - كرايشى) و اهمال

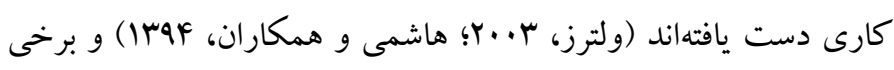

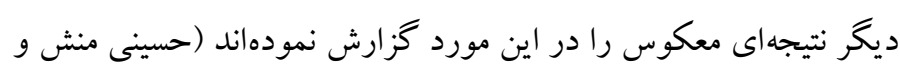

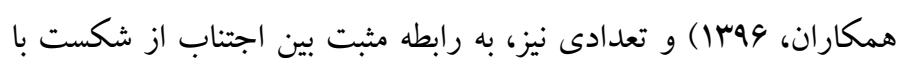


تضاد منافع: نو يسند كان هيج تضاد منافعى در رابطه با اين بزوهش اعلام نمىنمايند. تشكر و قدردانى: بدين وسيله از اساتيد راهنما و مشاوران خود و نيز كادر مدارس غير

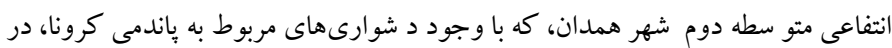
انجام اين تحقيق، از هيج كمكى مضايقه ننمودند، كمال تشكر و امتنان را دارم.
ملاحضات اخلاقى ييروى از ا صول اخلاق يزوهش: اين مقاله برگرفته از ر ساله دكترى نويسنده اول، در ر شته روانشنا سى تربيتى در دان شكده روانشنا سى و علومتربيتى داذشگاه خوارزمى،

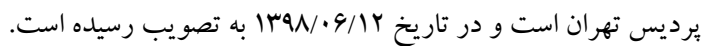
حامى مالى: اين ئزوهش در قالب رساله دكترى و بدون حمايت مالى مىباشد. نقش هر يكك از نويسند كان: نويسنده اول محقق اصلى اين يُوهش است، نويسنده دوم و سوم اساتيد راهنماى اول و دوم هستند و نويسندكان جهارم و بنجم اساتيد مشاوره رساله مىباشند. 


\section{References}

Akinsola, M. K., Tella, A., \& Tella, A. (2007). Correlates of Academic Procrastination and Mathematics Achievement of University Undergraduate Students. Eurasia Journal of Mathematics, Science \& Technology Education, 3, 363-370. [Link]

Ames, C. (1992). Classrooms: Goals, structures, and student motivation. Journal of Educational Psychology, 84, 261-271. [Link]

Amiri, L., Ebrahimi Moghadam, H., Babakhani, N. (2019). Structural model of predicting academic engagement based on cognitive ability and socioeconomic status by mediating academic enthusiasm in students. Journal of psychological science. (74) 18, 215-222. (Persian) [Link]

Arabzadeh, M., Askari, F., Rezvan, A., panahi, S. (2020). The role of self-construal and $3 * 2$ achievement goal in predicting of anxiety statistic in student. Journal of psychological science. (83) 18, 2141-2147. (Persian) [Link]

Batres, I. (2011). The relationship of grit, subjective happiness and meaning in life on alternative education students' GPA and attendance. CA: University of La Verne. [Link]

Bazelais, P., Lemay, D. J., \& Doleck, T. (2016). How Does Grit Impact College Students' Academic Achievement in Science?. European Journal of Science and Mathematics Education, 4(1), 33-43. [Link]

Bowman, N.A., Hill, P.L., Denison, N., \& Bronkema, R. (2015). Keep on truckin' or stay the course? Exploring grit dimensions as differential predictors of educational achievement, satisfaction, and intentions. Social Psychology and Personality Science, 6, 639-645. [Link]

Casuso-Holgado MJ, Cuesta-Vargas AI, Moreno-Morales N, Labajos-Manzanares MT, Baon-Lopez FJ, VegaCuesta M. (2013). The association between academic engagement and achievement in health sciences students. BMC medical Education. 13:33. [Link]

Christensen,R., Gerald, K. (2014). Comparative measures of grit, tenacity and perseverance. International Journal of Learning, Teaching and Educational Research, 8(1). [Link]

Cross, T. M. (2014). The gritty: Grit and non-traditional doctoral student success. Journal of Educators Online, 11(3), n3. [Link]

Duchesne, S., Larose, S., Bei, F. (2017). Achievement Goals and Engagement With Academic Work in
Early High School: Does Seeking Help From Teachers Matter?. Early Adolescence. 1-31 [Link]

Datu, J. A. D. V., Jana, P. M., \& King, R. B. (2016). The successful life of gritty students: Grit leads to optimal educational and well-being outcomes in a collectivist context Singapore: Springer. The Psychology of Asian Learners (pp. 503-516). [Link]

Duckworth, A. (2016). Grit: the power of passion and perseverance. London: Vermilion. [Link]

Duckworth, A. L. P., C; Matthews, M D; Kelly, Dennis R. (2007). Grit: perseverance and passion for longterm goals. Journal of personality and social psychology, 92(6), 1087. [Link]

Duckworth, A. L., \& Quinn, P. D. (2009). Development and validation of the Short Grit Scale (GRIT-S). Journal of personality assessment, 91(2), 166-174. [Link]

Dweck CS. (1986). Motivational processes affecting learning. Journal of American Psychology. 41(10): 1040-8. [Link]

Eskreis-Winkler, L., Gross, J. J., \& Duckworth, A. (2018). Grit: Sustained self-regulation in the service of superordinate goals. In K. D. Vohs, \& R. F. Baumeister (Eds.). Handbook of self-regulation (pp. 380-395). New York, NY: The Guilford Press 3rd. [Link]

Eskreis-Winkler, L., Duckworth, A. L., Shulman, E. P., \& Beal, S. (2014). The grit effect: Predicting retention in the military, the workplace, school, and marriage. Frontiers in Psychology, 5, 36. [Link]

Elliot, A. J. (2005). A conceptual history of the achievement goal construct. In A. J. Elliot \& C. S. Dweck (Eds.), Handbook of competence and motivation (pp. 52-72). New York: Guilford. [Link]

Elliot, A. J., \& McGregor, H. A. (2001). A 2x2 achievement goal framework. Journal of Personality and Social Psychology, 80, 501-519. [Link]

Elliot, A. J., McGregor, H. A., \& Gable, S. (1999). Achievement goals, study strategies, and exam performance: A meditational analysis. Journal of Educational Psychology, 91(3), 549-563. [Link]

Elliot, A. J., Murayama, K., \& Pekrun, R. (2011). A 3× 2 achievement goal model. Journal of Educational Psychology, 103(3), 632. [Link]

Feldman, D. B., \& Dreher, D. E. (2012). Can hope be changed in 90 minutes? Testing the efficacy of a single-session goal-pursuit intervention for college students. Journal of Happiness Studies, 13, 745759. [Link] 
Fredricks, J.A., Blumenfeld, P.C. and Paris, A.H. (2004) School Engagement: Potential of the Concept, State of the Evidence. Review of Educational Research, 74(1): 59 -109. [Link]

Gorman, R. (2015). An Examination of Academic Grit in Urban High Schools. [Link]

Hashemi Razini, H., Mousavi Panah, M., Shiri, m. (2015). Relationship between achievement goals orientation and motivational beliefs with academic procrastination and academic self-handicapping. Biquarterly journal of educational and scholastic studies. 3(11). 67-79. (Persian)[Link]

Hodge, B., Wright, B., Bennett, P. (2017). The Role of Grit in Determining Engagement and Academic Outcomes for University Students. Research in Higher Education, 59 (4), 448-460. [Link]

Holbein, J.B., Hillygus, D.S., Gibson-Davis, C.M., Hill, D., \& Lendard, M.A. (2016). The development of students' engagement in school, community, and democracy. [Link]

Howell, A. J. \& Watson, D. C. (2007). Procrastination: Associations with achievement goal orientation and learning strategies. Personality and Individual Differences, 43, 167-178. [Link]

Howell, A. J., Buro, K. (2009). Implicit beliefs, achievement goals, and procrastination: A mediational analysis. Learning and Individual Differences. 19, 151-154. [Link]

Hosseini manesh, Z., Omidian, M., Barzegar, K., (2017). The role of achievement goals, epistemological beliefs and quality learning experiences for students' educational procrastination. Quarterly journal of new thoughts on education. 44, 53-70. (Persian) [link]

Ivcevic, Z., \& Brackett, M. (2014). Predicting school success: Comparing conscientiousness, grit, and emotion regulation ability. Journal of research in personality, 52, 29-36. [Link]

Jimerson, S.R., Campos, E. and Greif, J. (2003) Toward an understanding of definitions and measures of school engagement and related terms. The California School Psychologist, 8: 7- 27. [Link]

Jin, H., Wang, W., Lan, X. (2019). Peer attachment and academic procrastination in Chinese college students: A moderated mediation model of future time perspective and grit. Frontiers in psychology, 10, 2645. [Link]

Karlen, Y., Suter, F., Hirt, C., Merki, K. M. (2019). The role of implicit theories in students' grit, achievement goals, intrinsic and extrinsic motivation, and achievement in the context of a long-term challenging task. Journal of Learning and Individual Differences. 74. 101757. [Link]

Kim, K. R., and Seo, E. H. (2015). The relationship between procrastination and academic performance: a meta-analysis. Pers. Individ. Diff. 82, 26-33. [Link]

Liem, G. A. D. (2016). Academic and social achievement goals: Their additive, interactive, and specialized effects on school functioning. British Journal of Educational Psychology, 86, 37-56. [Link]

Liem, G. A. D., Lau, S., \& Nie, Y. (2008). The role of selfefficacy, task value, and achievement goals in predicting learning strategies, task disengagement, peer relationship, and achievement outcome. Contemporary Educational Psychology,33, 486512. [Link]

Mason, H. D. (2018). Grit and academic performance among first-year university students: A brief report. Journal of Psychology in Africa, 28(1), 66-68. [Link]

McGregor H, Elliot AJ. (2002). Achievement goal as Predictors of achievement-relevant processes prior to task engagement. J Educational Psychology; 94(2): 381-95. [Link]

Middleton, M. J., \& Midgley, C. (1997). Avoiding the demonstration of lack of ability: An underexplored aspect of goal theory. Journal of Educational Psychology, 89(4), 710-718. [Link]

Miller, R. B., Greene, B. A., Montalvo, G. P., Ravindran, B., \& Nichols, J. D. (1996). Engagement in academic work: The role of learning goals, future consequences, pleasing others, and perceived ability. Contemporary Educational Psychology, 21, 388-422. [Link]

Muenks, K., Wigfield, A., Yang, J. S., \& O'Neal, C. R. (2017). How true is grit? Assessing its relations to high school and college students' personality characteristics, self-regulation, engagement, and achievement. Journal of Educational Psychology. 109(5), 599-620. [Link]

Perez, M. (2015). Obtaining academic success: Nurturing grit in students. Journal of Interpersonal Relations, Intergroup Relations and Identity, 8(Hiver/Winter), 56 63. [Link]

Pintrich, P. R. (2000b). The role of goal orientation in selfregulated learning. In M. Boekaerts, P. R. Pintrich, \& M. Zeidner (Eds.), Handbook of self-regulation (pp. 452-502). San Diego, CA: Academic Press. [Link]

Rastegar, A. (2012). Structural model of the relationships of classroom structure perception, achievement 
goals, achievement emotions and academic engagement: A comparative study of the traditional and virtual educational system. [Ph.D. thesis]. Tehran Payam Noor University. (Persian)[Link]

Rastegar, A., Talebi, S., Seif., M.H. (2015). Presenting a model for predicting academic procrastination based on personality traits and achievement goals among nursing students. Yazd Medical Sciences Education Studies and Development Center. 4, 318332. (Persian) [Link]

Reeve, J. (2012) A Self-determination Theory Perspective on Student Engagement. In Christenson, S. L., Reschly, A. L. and Wylie, C. (2012) The Handbook of Research on Student Engagement. pp. 149 - 172. New York: Springer Science. [Link]

Reeve, J. (2013) How Students Create Motivationally Supportive Learning Environments for Themselves: The Concept of Agentic Engagement. Journal of Educational Psychology. 105(3): 579 - 595. [Link]

Robinson, W.L. (2015). Grit and demographic characteristics associated with nursing student course engagement (Doctoral dissertation, Indiana University Purdue University Indianapolis). Retrieved from. [Link]

Ruzek, E. A., Hafen, C. A., Allen, J. P., Gregory, A., Mikami, A. Y., \& Pianta, R. C. (2016). How teacher emotional support motivates students: The mediating roles of perceived peer relatedness, autonomy support, and competence. Learning and Instruction, 42, 95-103. [Link]

Samareh, S., Kezri Moghadam, N. (2016). Relationship between achievement goals and academic selfEfficacy; Mediation role of academic engagement. Bimonthly of Education Strategies in Medical Sciences;8 (6):13-20. (Persian)[link]

Scher SJ, Osterman NM.) 2002(Procrastination conscientiousness, anxiety, and goals: Exploring the measurement and correlates of procrastination among school-aged children. Psychol School; 39(4): 385-98. [Link]

Seligman, M. E. P., \& Csikszentmihalyi, M. (2000). Positive psychology: An introduction. American Psychologist, 55 (1), pp. 5-14. [Link]

Senko, C. (2016). Achievement goal theory: A story of early promises, eventual discords, and future possibilities. In K. R. Wentzel \& D. B. Miele (Eds.), Handbook of motivation at school (2nd ed., pp. 7595). New York, NY: Taylor \& Francis. [Link]

Shirzadi, M. M., Bodaghi, A., Mirzaei, H., Babadi akashe, N., (2021). The effectiveness of self-determination skills training on academic incivility and academic engagement. Journal of psychological science. (99) 20, 391-404. (Persian) [Link]

Siah, P.C., Hui Wen NG, A., Dharmaraj, E., Foo, C., Mee Tan, S., Wider, W. (2019). Grit personality as a mediator or moderator for the effects of internet addiction on procrastination. Journal of Institutional Research South East Asia. 17(2) [Link]

Skinner, E., Kindermann, T., Furrer, C. (2009). A motivational perspective on engagement and disaffection conceptualization and assessment of children's behavioral and emotional participation in academic activities in the classroom. Educational and Psychological Measurement, 69(3):493-525 [Link]

Snyder, C. R., Michael, S. T., \& Cheavens, J. S. (1999). Hope as a psychotherapeutic foundation of nonspecific factors, placebos, and expectancies. In M. A. Huble, B. Duncan, \& S. Miller (Eds.), Heart and soul of change (pp. 205-230). Washington, DC: American Psychological Association. [Link]

Steel P. (2007). The nature of procrastination: A metaanalytic and theoretical review of quintessential self-regulatory failure. Psychology Bulletin; 133(1): 65-94. [Link]

Steel, P., and Klingsieck, K. B. (2016). Academic procrastination: psychological antecedents revisited. Aust. Psychol. 51, 36-46. [Link]

Stewart, S. B. (2015). Grit and self-control as predictors of first-year student success. University of Southern Maine. [Link]

Von Culin, K. R., Tsukayama, E., \& Duckworth, A. L. (2014). Unpacking grit: Motivational correlates of perseverance and passion for long-term goals. Journal of Positive Psychology. 9(4), 306-312. [Link]

Wang, M \& Erdhiem, J, (2007). Does the five-factor model of personality relate to goal orientation? Personality and Individual Differences, 43, pp. 1493- 1505. [Link]

Washington, A. S. (2016). The Relationship Between Student Grit and Student Achievement. Baker University. [Link]

Wentzel, K.R. (2002) The contribution of social goalsetting to children's school adjustment. In Wigfield, A, and Eccles, J. (eds.) Development of Achievement Motivation. (pp. 221-246). New York: Academic Press. [Link]

Wolters CA. (2003). Understanding procrastination from a self-regulated learning perspective. J Education Psychology; 95(1): 179-87[Link] 
Wolters, C. A. (2004). Advancing achievement goal theory: Using goal structures and goal orientations to predict students' motivation, cognition, and achievement. Journal of Educational Psychology, 96, 236-250. [Link]

Wolters, C. A., \& Hussain, M. (2015). Investigating grit and its relations with college students' self-regulated learning and academic achievement. Metacognition and Learning, 10(3), 293-311. [Link]

Wolters, C. A. H., \& Maryam, (2015). Investigating grit and its relations with college students' selfregulated learning and academic achievement. Metacognition And Learning, 10(3), 293-311. [Link]

Wolters, C. A., Yu, S.L., \& Pintrich, P. R. (1996).The relation between goal orientation and students' motivational beliefs and self-regulated learning. Learning and Individual Differences, 8(3), 211238. [Link]

Zweig, D \& webester, J, (2004). What are we examining? An examination of the relationship between the bigfive personality traits, goal orientation, and performance intention, Personality, and Individual Differences, 36, pp. 1693-1708. [Link] 